“ (C) 2018 IEEE. Personal use of this material is permitted. Permission from IEEE must be obtained for all other uses, in any current or future media, including

reprinting/republishing this material for advertising or promotional purposes, creating new collective works, for resale or redistribution to servers or lists, or reuse of any copyrighted component of this work in other works." 


\title{
Wideband Dual-Polarized Multiple Beam-Forming Antenna Arrays
}

\author{
He Zhu, Hai-Han Sun, Bevan Jones, Life Member, IEEE, Can Ding, and Y. Jay Guo, Fellow, IEEE
}

\begin{abstract}
Novel wideband multi-beam antenna arrays based on wideband Butler matrices are presented in this paper. The proposed beam-forming arrays are particularly suited to increasing the capacity of 4G long-term evolution (LTE) base stations. Although dual-polarized arrays are widely used in LTE base stations, analogue beam-forming arrays have not been realized before, due to the huge challenge of achieving wide operating bandwidth and stable array patterns. To tackle these problems, for the first time, we present a novel wideband multiple beam-forming antenna array based on Butler matrices. The described beam-forming networks produce three beams but the methods are applicable to larger networks. The essential part of the beam-forming array is a wideband three-beam Butler matrix, which comprises quadrature couplers and fixed wideband phase shifters. Wideband quadrature and phase shifters are developed using striplines, which provide the required power levels and phase differences at the outputs. To achieve the correct beamwidth and to obtain the required level of crossover between adjacent beams, beam-forming networks consisting of augmented three-beam Butler matrices using power splitters are presented to expand the number of output ports from three to five or six. Dual-polarized, three-beam antenna arrays with 5 and 6 elements covering LTE band are developed. Prototypes comprising beam-forming networks and arrays are tested according to LTE base station specification. The test results show close agreement with the simulation ones and compliance with LTE requirements. The designs presented are applicable to a wide range of wideband multi-beam arrays.
\end{abstract}

Index Terms -Antenna arrays, beam-forming networks, Butler matrix, dual-linear polarization, LTE base stations, multi-beam, phase shifters, quadrature couplers, wideband.

\section{INTRODUCTION}

$\mathrm{C}$ ROWDED data traffic is a major issue to tackle in the current wireless communication systems, especially in the 4G LTE base stations where high data rates are needed. Antenna features such as high gain, wide bandwidth and high directivity are important and highly desired to meet the system requirements [1]-[4]. Linear arrays have been widely used in $2 \mathrm{G}, 3 \mathrm{G}$, or LTE base stations to achieve these characteristics [5]-[10]. Dual-polarized antenna arrays consisting of 4 and 8 elements achieved high gain of $14 \mathrm{dBi}$ and $16 \mathrm{dBi}$ in [4] and [6], respectively. By interleaving high- and low-band elements,

Manuscript submitted August 9, 2018; revised .

The authors are with the Global Big Data Technologies Centre (GBDTC), University of Technology Sydney, Ultimo, Sydney, NSW 2007, Australia (email: he.zhu@uts.edu.au) dual-band dual-polarized antenna arrays were designed in [6]-[9]. High gain and directivity were obtained in these and many similar arrays by employing vertical arrays (columns). In order to increase system capacity, base stations with multiple columns are widely used in MIMO configurations [11]-[12].

Another way to increase capacity is using multiple beams to create more cells. In the areas of high traffic density such as concert arenas and sports stadiums, single-sector antennas may not provide sufficient capacity and it is desirable to divide the coverage area into a number of smaller cells served from a single point. In this case, antennas or antenna arrays with multiple beams covering multiple sectors can provide capacity advantages over single-sector antennas. Multi-beam antennas that provide horizontally sectorized multiple-cell coverage within the areas for a particular event are of great interest. Another application of multi-beam antennas is in the traditional three-sector cellular base stations where one or more of the sectors with high traffic density can be divided into sub-sectors with separate cells to increase capacity. In all these cases, a wideband analog beam-forming network serves as a key technology.

Typical beam-forming networks include Butler matrix, Blass matrix and Nolen matrix, among which Butler matrix [13] is the most commonly used as it is simple and can be lossless in principle. Butler matrices have been employed in various smart antenna systems [14]-[17]. Most recently, there has been an increasing interest in Butler matrices, and various fabrication technologies such as low-temperature co-fired ceramic (LTCC) [18], waveguide [19] and substrate integrated waveguide (SIW) [20]-[22], have been used to implement Butler matrices to millimeter-waves systems for $5 \mathrm{G}$ mobile or fixed wireless access communication. Various techniques for circuit miniaturization [23]-[24], bandwidth enhancement [25], and beams control [26] have been applied to traditional $4 \times 4$ Butler matrices, to obtain more attractive features. Traditional uniform Butler matrices usually include $2^{n}$ input ports and $2^{n}$ output ports, such as $2 \times 2,4 \times 4$ and $8 \times 8$ formats. In many cases, however, $M \times N$ beam-forming networks are more desirable where the number of beams is not constrained to $2^{n}$. For cell sectorization, it is much more economical to choose the required number of beams based on the coverage requirements rather than needing to use $2^{n}$ elements as adding extra cells is costly if they are not needed. Some non-uniform beam-forming networks have been described such as in [27]-[29]; however, some of them are narrow-band solutions and none of them has been applied to wideband wireless communication systems like LTE base stations. 
In LTE base stations, arrays with multiple vertical columns of dual polarization elements are arranged horizontally to achieve a narrow beam in the elevation direction and multiple beams in the azimuth direction, thereby meeting the need for large data capacity. Usually, a single beam antenna provides coverage over a $120^{\circ}$ sector in the azimuth plane whereas a multi-beam antenna covers the same sector with multiple narrower beams corresponding to separate cells. In this case, the data capacity is dramatically increased due to the increased number of cells. For instance, a two-beam array integrated with a $2 \times 4$ Butler matrix was described in [30] for covering a one-sector of the traditional tri-sector base station to double the capacity. However, though the beam number is doubled, two beams may still not provide enough data capacity in some high-density areas. High performance multi-beam networks such as those presented here are essential for expanding the capacity of existing systems as population density increases, and single-beam antennas are a huge limitation in extending the data capacity of LTE systems. Therefore, there is an urgent industrial need to develop new solutions to build wideband multi-beam arrays in wireless communication systems.

In this paper, we present the design of a wideband Butler matrix based beam-forming network, which covers the LTE band from $1.71 \mathrm{GHz}$ to $2.69 \mathrm{GHz}$. The wideband beamforming network is employed in two alternative beam-forming arrays. The challenges are mainly from the requirements for wide operating bandwidth, pattern stability and sidelobe levels, and in an environment of strong mutual coupling within arrays. Considering the existing shortcomings and challenges in the current LTE base stations, this paper has achieved the following contributions: (1) a wideband three-beam beamforming network to cover the entire LTE band with flexible beamwidth; (2) new solutions for the design of key components in the beam-forming networks including wideband quadrature couplers, phase shifters and power dividers; (3) dual-polarized antenna arrays using compact elements with stable patterns and steerable beams that meet LTE base station criteria; and (4) excellent antenna array performance based on a reduced size element that minimizes coupling; (5) the beam-forming performance extends across the whole $4 \mathrm{G}$ band and have been verified by experiment.

The paper is organized as follow. Section II describes the configurations of beam-forming arrays to meet LTE base station criteria. Section III considers the theory of three-beam Butler matrix, based on which wideband components, including quadrature couplers and phase shifters are presented, modeled and simulated. Section IV gives thorough descriptions of wideband beam-forming networks that are used in LTE base stations to feed dual-polarized arrays. Section V presents the radiating element as well as arrays used in the multi-beam arrays for validating the proposed beam-forming networks. Section VI shows all the simulated and tested results of beam-forming arrays, confirming all the designs. Finally, section VII concludes the paper.

\section{BEAM-Forming NETWORKS FOR LTE BASE StATIONS}

LTE base station antennas with multiple beams usually

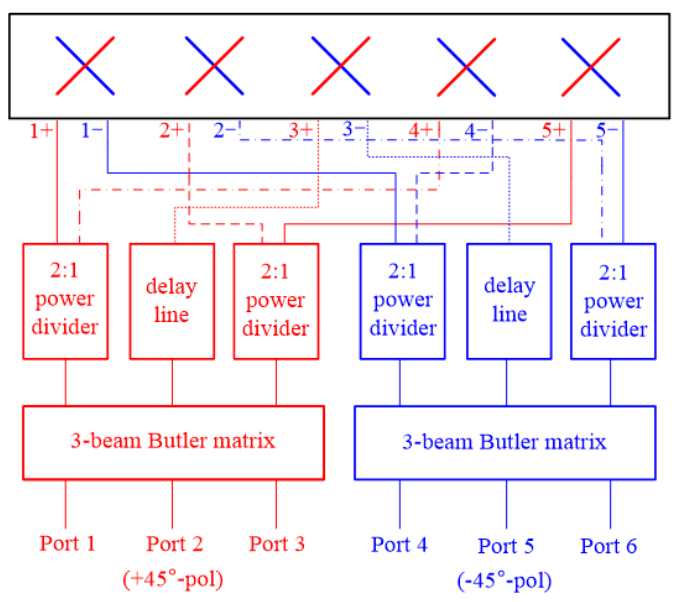

(a)

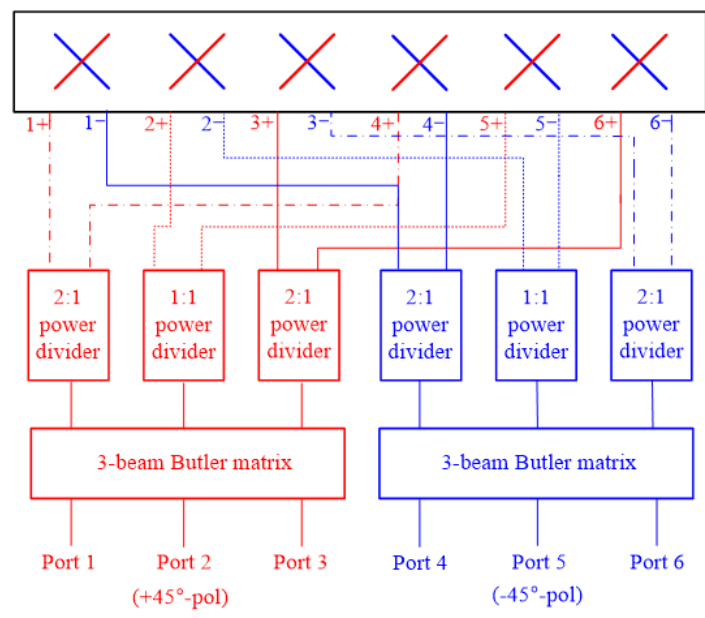

(b)

Fig. 1. Configurations of two beam-forming arrays for LTE base stations: (a) using 5-element array; (b) using 6-element array.

Table I

Power and Phase Distribution on the 5-Element Array

\begin{tabular}{c|c|ccccc}
\hline \hline \multicolumn{2}{c|}{ Element No. } & 1 & 2 & 3 & 4 & 5 \\
\hline \multicolumn{2}{c}{ Power Level } & $1 / 3$ & $2 / 3$ & 1 & $2 / 3$ & $1 / 3$ \\
\hline \multicolumn{2}{c}{ Amplitude (dB) } & -4.77 & -1.77 & 0 & -1.77 & -4.77 \\
\hline \multirow{2}{*}{ Phase } & Port 1 & 0 & -120 & 120 & 0 & -120 \\
$\left(^{\circ}\right)$ & Port 2 & 0 & 120 & -120 & 0 & 120 \\
& Port 3 & 0 & 0 & 0 & 0 & 0 \\
\hline \hline
\end{tabular}

Table II

Power and Phase Distribution on the 6-Element Array

\begin{tabular}{c|c|cccccc}
\hline \hline \multicolumn{2}{c|}{ Element No. } & 1 & 2 & 3 & 4 & 5 & 6 \\
\hline \multicolumn{2}{c}{ Power Level } & $1 / 3$ & $1 / 2$ & $2 / 3$ & $2 / 3$ & $1 / 2$ & $1 / 3$ \\
\hline \multicolumn{2}{c}{ Amplitude (dB) } & -4.77 & -3 & -1.77 & -1.77 & -3 & -4.77 \\
\hline \multirow{2}{*}{ Phase } & Port 1 & 0 & -120 & 120 & 0 & -120 & 120 \\
$\left({ }^{\circ}\right)$ & Port 2 & 0 & 120 & -120 & 0 & 120 & -120 \\
& Port 3 & 0 & 0 & 0 & 0 & 0 & 0 \\
\hline \hline
\end{tabular}

consist of beam-forming networks and an array of dual-linearly polarized antenna columns. In this work, single elements are used in place of columns in order to study beam-forming in the azimuth plane. The beam-forming networks divide the coverage area in the azimuth plane into $n$ (in our case three) parts. If the coverage sector is of angular width $\alpha$, then each 
beam should cover a sector of $(\alpha / n)$. In LTE base stations, three arrays are typically arranged to cover $360^{\circ}$ arc and thus $\alpha$ is $120^{\circ}$ for each array. The crossover point is defined as the intersection point of two adjacent beams, which may have different levels relative to the beam peak depending on the array configurations. Basic Butler matrices are such that if the outputs are connected to omnidirectional elements, the beam crossovers occur at $-3 \mathrm{~dB}$. For LTE multi-beam antennas the crossover level should normally be at about $-10 \mathrm{~dB}$ so, to achieve this, beams must be made narrower which can be achieved if the array is lengthened.

To this end, we extend the arrays from 3 elements to 5 or 6 elements as shown in Fig. 1, which shows block diagrams of the 5- and 6-element realizations. We refer to such a beamforming network as an augmented Butler matrix. This achieves beam crossover values in the range from $-10 \mathrm{~dB}$ to $-15 \mathrm{~dB}$, which is suitable for LTE applications. Here we intend to generate three beams in the azimuth plane to cover the $120^{\circ}$ sector with three separate beams each covering a sector of $40^{\circ}$. Each beam-forming array is composed of two three-beam Butler matrices, power dividers/delay lines and a dual-linearly polarized array. Apart from its ability to control the crossover level between the beams, the augmented Butler matrix necessarily provides an amplitude taper across the array aperture which results in a reduced sidelobe level. Some control of the sidelobe level can be exercised by selecting the split ratio of the power dividers. A suitable arrangement of a 5- and 6-element beam-forming arrays are shown in Fig. 1. Table I and II display the ideal power level and phase distribution on the two arrays.

The three-beam Butler matrix is the essential part of the beam-forming network. To produce three beams, phase increments of $0^{\circ}, 120^{\circ}$ and $-120^{\circ}$ are required between the outputs for the three required beams. These fixed phase relationships are required over the whole operating band. The Butler matrix is composed of quadrature couplers and fixed phase shifters. All of these components are required to be wideband devices. Fig. 2 shows how a three-way Butler matrix with the correct output amplitudes and phases can be constructed from quadrature couplers and fixed phase shifters. For each antenna element in the LTE base station, $\pm 45^{\circ}$ polarization is required and separate beam-forming networks and dual-polarized radiating elements are needed for the two polarizations. In the cellular environment, the three inputs to the Butler matrix correspond to three adjacent beams and are fed with signals for three separate cells. The single multi-beam antenna is used as an alternative to three independent antennas with different pointing fed from three separate cells.

\section{Design OF Wideband ThreE-BEAm ButLer MATRIX}

In this section, we provide the theory and engineering design of the essential components within the proposed wideband beam-forming network.

\section{A. Theory of the Three-beam Butler Matrix}

For a three-beam beam-forming network with perfect port matching and ideal isolation, the scattering matrix of the three-

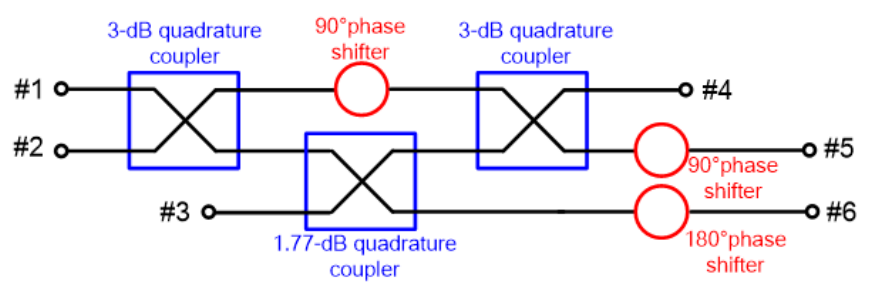

Fig. 2. Configuration of the proposed wideband three-beam Butler matrix for LTE base stations.

beam Butler matrix with ports 1,2 and 3 as inputs and port 4,5 and 6 as outputs can be expressed as:

$$
S=\left[\begin{array}{ll}
0 & T \\
T & 0
\end{array}\right]
$$

where

$$
T=\frac{1}{\sqrt{3}}\left[\begin{array}{ccc}
e^{-j \pi} & e^{j \frac{\pi}{3}} & e^{-j \frac{\pi}{3}} \\
e^{-j \pi} & e^{-j \frac{\pi}{3}} & e^{j \frac{\pi}{3}} \\
e^{-j \pi} & e^{-j \pi} & e^{-j \pi}
\end{array}\right]
$$

The matrix of the transmission block of $S$, is a symmetrical unitary matrix. From (2) one can observe the phase differences between any two adjacent elements of each row are $-\frac{2}{3} \pi, \frac{2}{3} \pi$ and 0 , respectively. To construct such a beam-forming network, quadrature couplers and fixed phase shifters are required to provide appropriate power division and phase at each output port. The quadrature coupler can be represented with an orthogonal transmission matrix $Q$, which is written as:

$$
Q=\left[\begin{array}{cc}
a & -j \sqrt{1-a^{2}} \\
-j \sqrt{1-a^{2}} & a
\end{array}\right]
$$

where $a$ is the signal magnitude at one output port, and the signal magnitude at the other port is $\sqrt{1-a^{2}}$. There is $90^{\circ}$ phase difference between two output ports due to the orthogonal property of the quadrature coupler. For the equalsplit quadrature coupler $Q_{1}$ with power division ratio of 1:1 $(a=1 / \sqrt{2})$ and unequal-split quadrature coupler $Q_{2}$ with power division ratio of $2: 1 \quad(a=1 / \sqrt{3})$, the transmission matrices become, respectively:

$$
\begin{aligned}
& Q_{1}=\left[\begin{array}{cc}
\frac{1}{\sqrt{2}} & -j \frac{1}{\sqrt{2}} \\
-j \frac{1}{\sqrt{2}} & \frac{1}{\sqrt{2}}
\end{array}\right] \\
& Q_{2}=\left[\begin{array}{cc}
\frac{1}{\sqrt{3}} & -j \frac{\sqrt{2}}{\sqrt{3}} \\
-j \frac{\sqrt{2}}{\sqrt{3}} & \frac{1}{\sqrt{3}}
\end{array}\right]
\end{aligned}
$$

The transmission block $T$ can be written as:

$$
T=P_{1} T_{1} P_{2}
$$

where

$$
T_{1}=\left[\begin{array}{ll}
Q_{1} & \\
& 1
\end{array}\right]\left[\begin{array}{ll}
-j & \\
& Q_{2}
\end{array}\right]\left[\begin{array}{ll}
Q_{1} & \\
& 1
\end{array}\right]
$$




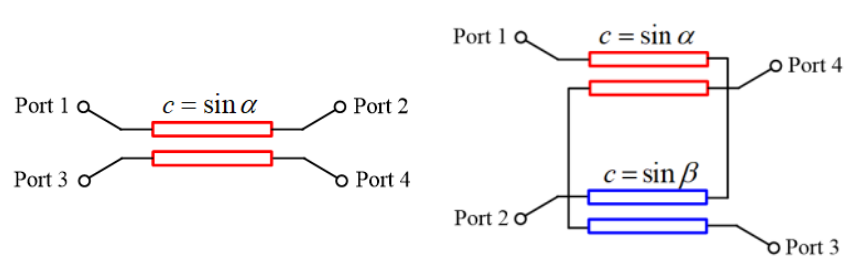

(a)

(b)

Fig. 3. Equivalent transmission line models of two quadrature couplers: (a) traditional type; (2) tandem type.

$$
\begin{gathered}
P_{1}=\left[\begin{array}{lll}
e^{-j \frac{\pi}{3}} & & \\
& e^{-j \frac{\pi}{6}} & \\
& & 1
\end{array}\right] \\
P_{2}=\left[\begin{array}{lll}
1 & & \\
& -j & \\
& & -1
\end{array}\right]
\end{gathered}
$$

Here, $T_{1}, P_{1}$ and $P_{2}$ are all diagonal matrices: $T_{1}$ is the center part of $T$ composed of three components: two equal-power quadrature couplers $Q_{1}(a=1 / \sqrt{2})$ and one unequal-power quadrature coupler $Q_{2}(a=1 / \sqrt{3})$ with a $90^{\circ}$ phase shifter. $P_{1}$ and $P_{2}$ refer to two phase shifting networks, by which $P_{1}$ will add phase shifts on the row elements while $P_{2}$ will add phase shifts on the column elements of $T_{1}$.

In (2), the phases of the first elements of each row are identical. However, it makes no difference what this value is, which means the phase shift block $P_{1}$ in (5) can be neglected, and the transmission block $T$ can be simplified to $T_{r}$ as:

$$
T_{r}=T_{1} P_{2}=\frac{1}{\sqrt{3}}\left[\begin{array}{ccc}
e^{-j \frac{2 \pi}{3}} & e^{j \frac{2 \pi}{3}} & 1 \\
e^{-j \frac{5 \pi}{6}} & e^{-j \frac{\pi}{6}} & e^{j \frac{\pi}{2}} \\
e^{-j \pi} & e^{-j \pi} & e^{-j \pi}
\end{array}\right]
$$

It is seen that the phase differences between adjacent elements in each row are $-\frac{2}{3} \pi, \frac{2}{3} \pi$ and 0 , respectively, which satisfy the phase requirements of a three-beam Butler matrix. Based on the analysis above, the three-beam Butler matrix can be configured as shown in Fig. 2. The configuration is quite simple as it is composed of two $3-\mathrm{dB}$ and one $1.77-\mathrm{dB}$ quadrature couplers, and three phase shifters with $90^{\circ}$ and $180^{\circ}$ phase shifts. Since the design is aimed at LTE band range from $1.71 \mathrm{GHz}$ to $2.69 \mathrm{GHz}$, wideband components operating over this band need to be developed accordingly.

\section{B. Quadrature Couplers}

Following the analysis above, we aim to develop a new type of quadrature couplers that can realize equal and unequal coupling power ratio with wide operating band range. To satisfy the requirements of the bandwidth and power ratio, directional couplers with coupled-lines using striplines are investigated. Fig. 3(a) shows the configuration of a traditional backward wave directional coupler with four ports. The overall length of the coupler is $90^{\circ}$ at the center frequency, and the coupling factor of the coupled-line section is $c$. As mentioned in the last section, the output amplitude at two ports is $a$ and $\sqrt{1-a^{2}}$, and if we write $c=\sin \alpha$, the scattering matrix of the

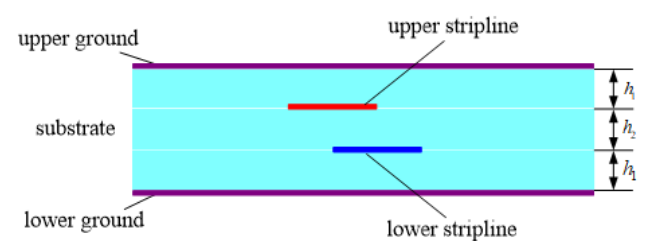

(a)
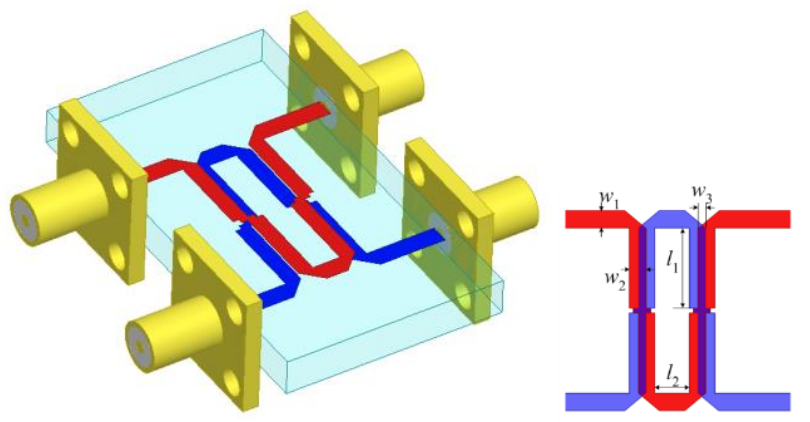

(b)

Fig. 4. Configuration of (a) Cross-sectional view of the stripline implementation used in the design of quadrature coupler; (b) 3D model of the constructed quadrature coupler.

TABLE III

RELATED PARAMETERS OF TWO QUADRATURE COUPLERS

\begin{tabular}{cccc}
\hline \hline \multicolumn{2}{c}{3 -dB coupler } & \multicolumn{2}{c}{ 1.77-dB coupler } \\
Parameters & Values $(\mathrm{mm})$ & Parameters & Values $(\mathrm{mm})$ \\
\hline$l_{1}$ & 9.2 & $l_{1}$ & 8.8 \\
$l_{2}$ & 3.0 & $l_{2}$ & 3.0 \\
$w_{1}$ & 1.96 & $w_{1}$ & 1.96 \\
$w_{2}$ & 1.5 & $w_{2}$ & 1.52 \\
$w_{3}$ & 0.24 & $w_{3}$ & 0.76 \\
\hline \hline
\end{tabular}

coupler $S_{c 1}$ becomes [31]:

$$
S_{c 1}=\left[\begin{array}{cccc}
0 & \cos \alpha & -j \sin \alpha & 0 \\
\cos \alpha & 0 & 0 & -j \sin \alpha \\
-j \sin \alpha & 0 & 0 & \cos \alpha \\
0 & -j \sin \alpha & \cos \alpha & 0
\end{array}\right]
$$

where $\alpha$ is an angle depending on the power division ratio (PDR) of the coupler. For a 3-dB coupler when the PDR equals to $1: 1, \cos \alpha=\sin \alpha=1 / \sqrt{2}$ and $\alpha=45^{\circ}$; for a $1.77-\mathrm{dB}$ coupler when the PDR equals to $2: 1, \cos \alpha=1 / \sqrt{3}, \sin \alpha=$ $\sqrt{2} / \sqrt{3}$ and $\alpha=54.74^{\circ}$. Ports 2 and 3 refer to the direct port and coupled port, where there is $90^{\circ}$ phase difference between them; hence the term "quadrature coupler".

For an unequal directional coupler with a PDR of $2: 1$, the coupling factor is about 0.82 , which is so high that it is difficult to realize using planar structures. To tackle the problem, a tandem structure shown in Fig. 3(b) is used. Assuming that the coupling factors of the two coupled-line sections are $\alpha$ and $\beta$, the output signal magnitude in this case can be calculated as:

- $\quad$ port 2: $\cos \alpha \cos \beta-\sin \alpha \sin \beta=\cos (\alpha+\beta)$

- $\quad$ port 3: $-j \cos \alpha \sin \beta-j \sin \alpha \cos \beta=-j \sin (\alpha+\beta)$

Therefore, the scattering matrix of the cascaded quadrature coupler $S_{c 2}$ can be written as: 


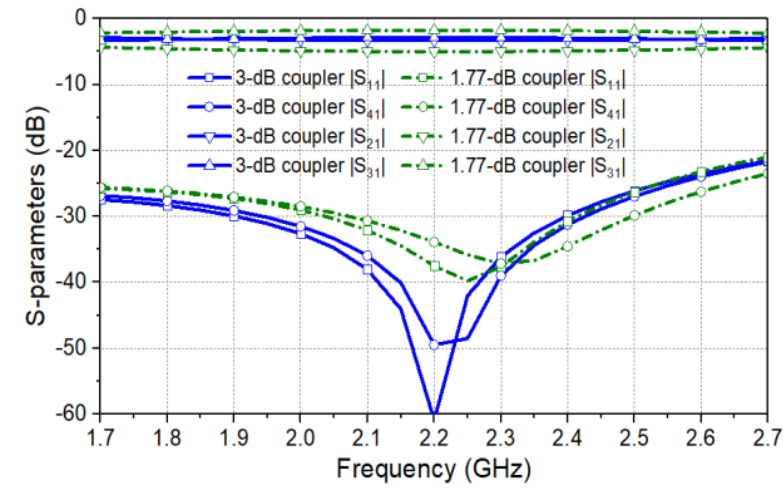

(a)

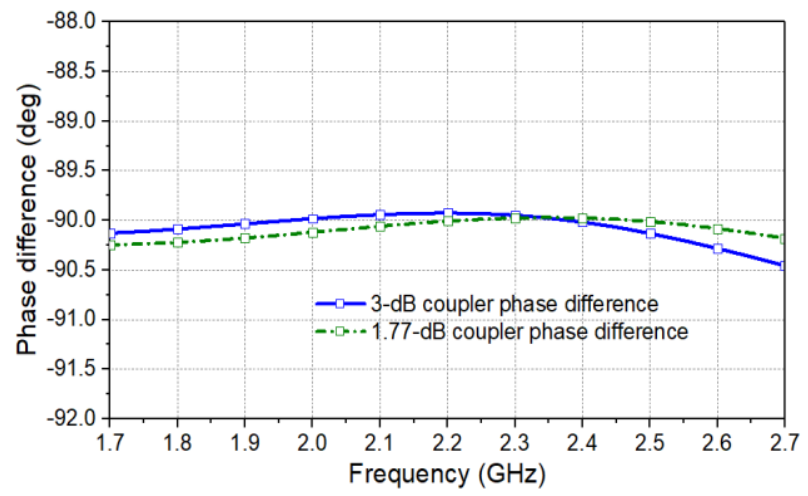

(b)

Fig. 5. Simulated reflection coefficients of the antenna with ideal circuit feed network and its physical realization.

$$
=\left[\begin{array}{cccc}
S_{c 2} & & & \\
0 & \cos (\alpha+\beta) & -j \sin (\alpha+\beta) & 0 \\
\cos (\alpha+\beta) & 0 & 0 & -j \sin (\alpha+\beta) \\
-j \sin (\alpha+\beta) & 0 & 0 & \cos (\alpha+\beta) \\
0 & -j \sin (\alpha+\beta) & \cos (\alpha+\beta) & 0
\end{array}\right]
$$

If $\alpha=\beta$, the scattering matrix can be simplified as:

$$
S_{c 2}=\left[\begin{array}{cccc}
0 & \cos 2 \alpha & -j \sin 2 \alpha & 0 \\
\cos 2 \alpha & 0 & 0 & -j \sin 2 \alpha \\
-j \sin 2 \alpha & 0 & 0 & \cos 2 \alpha \\
0 & -j \sin 2 \alpha & \cos 2 \alpha & 0
\end{array}\right]
$$

Compared (10) with (8), it is found that the two-stage cascaded structure is able to realize a coupling factor that is twice that of the single stage coupler for the same degree of coupling of the lines. The even- and odd-mode impedance can be calculated by the following equations:

$$
\begin{aligned}
& Z_{0 e}=Z_{0} \sqrt{\frac{1+c}{1-c}} \\
& Z_{0 o}=Z_{0} \sqrt{\frac{1-c}{1+c}}
\end{aligned}
$$

In the cascaded structure, the coupling factor of the coupled-line is half that of the overall coupler. Therefore, for the $3-\mathrm{dB}$ coupler, the even- and odd-mode impedances are $74.8 \Omega$ and $33.4 \Omega$; for the 1.77-dB coupler, the even- and odd-mode impedances are $82.2 \Omega$ and $30.4 \Omega$, respectively. All ports of the quadrature couplers should be matched at all

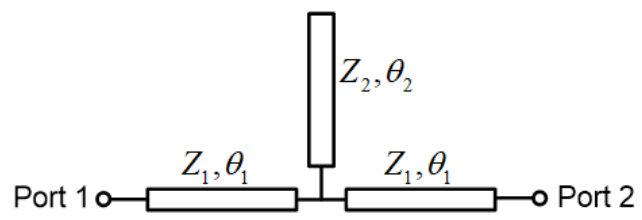

(a)

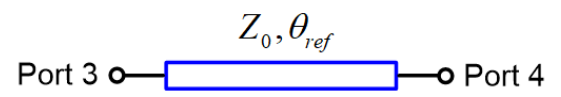

(b)

Fig. 6. Equivalent transmission line models of the wideband phase shifter: (a) phase shifter; (b) reference line.

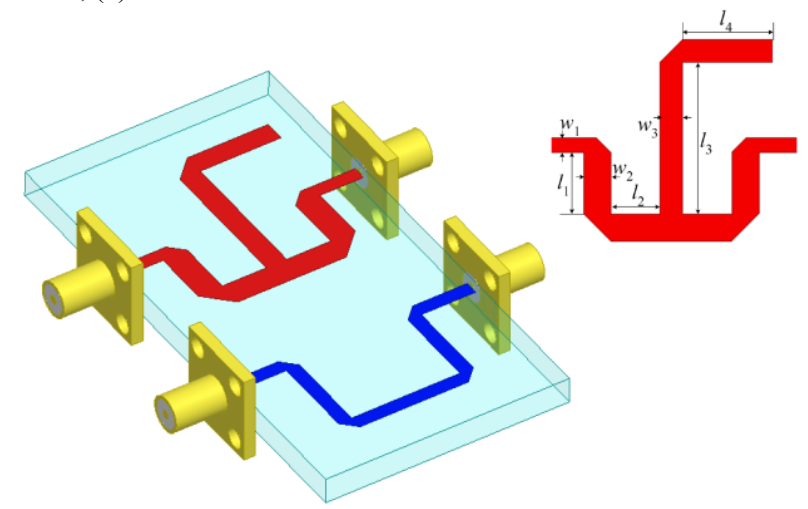

Fig. 7. 3D model of the constructed phase shifter.

frequencies, requiring that the even and odd mode velocities are equal across the band. Microstrip construction has unequal velocities and can only be approximately compensated [32]. Stripline inherently has equal even and odd mode velocities and normally provides much better performance where coupled lines are required. Fig. 4(a) shows the cross-section view of the proposed stripline, which has three stacked substrate boards each with dielectric constant of $\varepsilon_{r}$ and thickness of $h_{1}, h_{2}$ and $h_{1}$, respectively. There are metal layers on the top and bottom of the substrates acting as grounds. Broadside coupling occurs between the upper and lower striplines, which are located on two sides of the middle substrate.

Fig. 4(b) shows the 3D structure of the quadrature coupler. In this work, two different couplers with coupling coefficient of 3-dB and $1.77 \mathrm{~dB}$ are built in Ansys EM Desktop, and the relevant dimensions of two couplers are listed in Table III. The coupling coefficient can be controlled by changing the line width as well as the overlap between the upper and lower stripline. The substrate used for the design is Rogers RO4356B with $\varepsilon_{r}$ of 3.48 and $h_{1}, h_{2}$ equal to $1.52 \mathrm{~mm}$ and $0.76 \mathrm{~mm}$, respectively.

Full-wave simulation results are shown in Fig. 5. Fig. 5(a) shows that the power magnitude at port 2 and port 3 of two quadrature couplers with PDR of $1: 1$ and $2: 1$. For the equal PDR case, $\left|S_{21}\right|$ and $\left|S_{31}\right|$ is about $-3.1 \pm 0.3 \mathrm{~dB}$ across the frequency range from $1.7 \mathrm{GHz}$ to $2.7 \mathrm{GHz}$ compared with the ideal power level $-3 \mathrm{~dB}$. For the unequal coupler, $\left|S_{21}\right|$ and $\left|S_{31}\right|$ is about $-1.9 \pm 0.2 \mathrm{~dB}$ and $-4.7 \pm 0.3 \mathrm{~dB}$, which is very close to the ideal value of $-1.77 \mathrm{~dB}$ and $-4.77 \mathrm{~dB}$. Fig. 5(b) demonstrates 


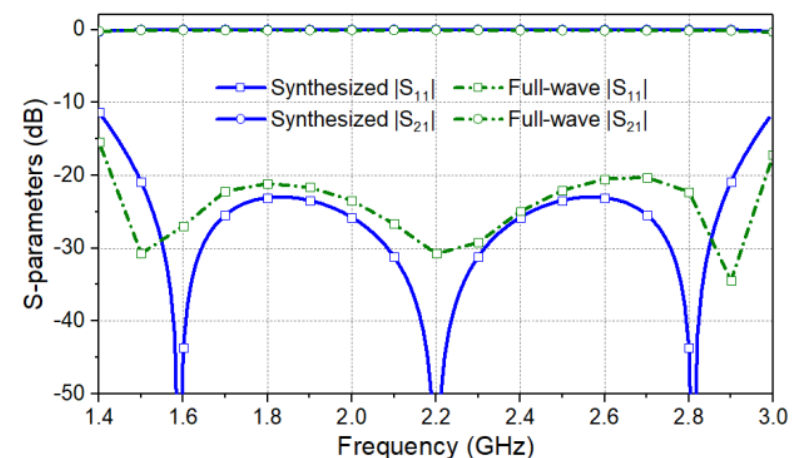

(a)

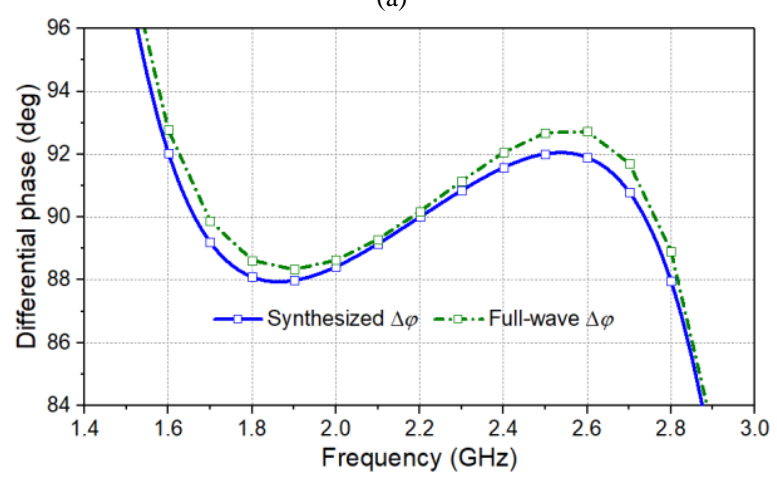

(b)

Fig. 8. Synthesized and full-wave simulation results of the phase shifter: (a) S-parameters; (b) differential phase.

that both designs achieve excellent port matching and isolation across the whole band. Moreover, the phase difference between two output ports is constant and stable around $90^{\circ}$, representing the orthogonality property of the quadrature couplers.

\section{Wideband Fixed Phase Shifters}

In addition to wideband quadrature couplers, fixed phase shifters are also critical components in the Butler matrix. To achieve stable phase, excellent matching and transmission property and small phase deviation are extremely important. To that end, a novel type of wideband phase shifter is designed. Fig. 6(a) shows the equivalent circuit of a suitable phase shifter. It comprises two transmission lines $\left(Z_{1}, \theta_{1}\right)$ and an open-ended stub $\left(Z_{2}, \theta_{2}\right)$ at the centre point of two transmission lines. A reference line, as shown in Fig. 6(b), is required for comparison with the phase shifter section. The operating principle of the phase shifter is that if the inputs of the phase shifter and reference line (port 1 and port 3 in Fig. 6) are fed with equal, in-phase signals, the outputs maintain an almost constant phase difference across the band. The ABCD matrix of the phase shifter can be calculated by:

$$
\begin{aligned}
& {[A]=\left[\begin{array}{cc}
\cos \theta_{1} & j Z_{1} \sin \theta_{1} \\
j \frac{1}{z_{1}} \sin \theta_{1} & \cos \theta_{1}
\end{array}\right] \cdot\left[\begin{array}{cc}
1 & 0 \\
j \frac{1}{z_{2}} \tan \theta_{2} & 1
\end{array}\right] } \\
& \cdot\left[\begin{array}{cc}
\cos \theta_{1} & j Z_{1} \sin \theta_{1} \\
j \frac{1}{Z_{1}} \sin \theta_{1} & \cos \theta_{1}
\end{array}\right]
\end{aligned}
$$

Subsequently, using the ABCD-to-S-matrix conversion, the $\mathrm{S}$-parameters of the phase shifter are derived as:

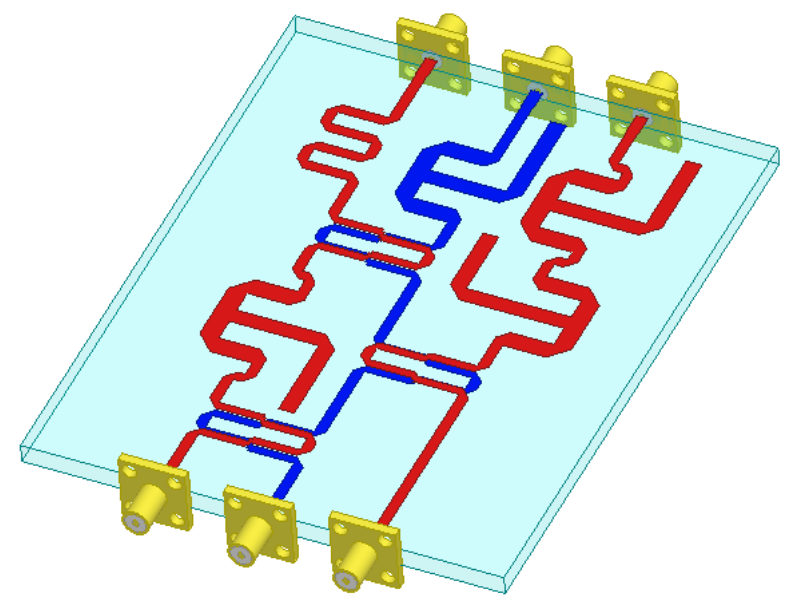

(a)

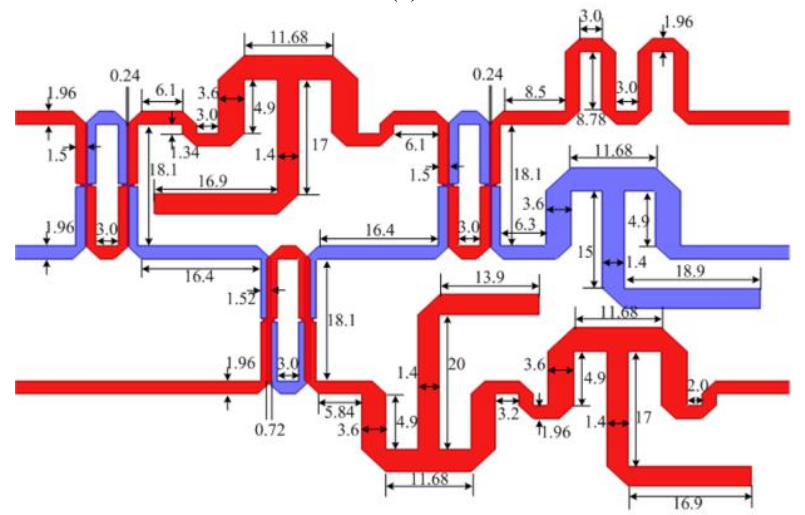

(b)

Fig. 9. 3D model of the proposed 3-beam Butler matrix for LTE base stations.

$$
\begin{aligned}
S_{11} & =\frac{B-C Z_{o}^{2}}{2 Z_{o} A+B+C Z_{o}^{2}} \\
S_{21} & =\frac{2 Z_{o}}{2 Z_{o} A+B+C Z_{o}^{2}}
\end{aligned}
$$

where

$$
\begin{aligned}
A & =\cos \left(2 \theta_{1}\right)-\frac{Z_{1}}{2 Z_{2}} \sin \left(2 \theta_{1}\right) \cdot \tan \theta_{2} \\
B & =j \cdot\left[Z_{1} \sin \left(2 \theta_{1}\right)-\frac{Z_{1}^{2}}{Z_{2}} \sin ^{2} \theta_{1} \cdot \tan \theta_{2}\right] \\
C & =j \cdot\left[\frac{1}{Z_{1}} \sin \left(2 \theta_{1}\right)-\frac{1}{Z_{2}} \cos ^{2} \theta_{1} \cdot \tan \theta_{2}\right]
\end{aligned}
$$

The differential phase shift can be calculated by comparing the phase of the proposed structure with that of the reference line, which is expressed as:

$$
\begin{aligned}
\Delta \phi & =\angle S_{21 \_p s}-\angle S_{21_{r e f}} \\
& =\theta_{r}-\tan ^{-1}\left[\frac{B+C Z_{o}^{2}}{2 j \cdot Z_{o} A}\right]
\end{aligned}
$$

For an ideal wideband phase shifter, it is required to have $\left|S_{21}\right|=1,\left|S_{11}\right|=0$, and $\Delta \phi=\theta_{r}-180^{\circ}$. In this case, the related value can be determined as: $\theta_{1}=90^{\circ}$ and $\theta_{2}=180^{\circ}$. These phase shifters can achieve a wide and constant differential phase across the desired operating band. To obtain $90^{\circ}$ phase shift, the length of the reference line should be 


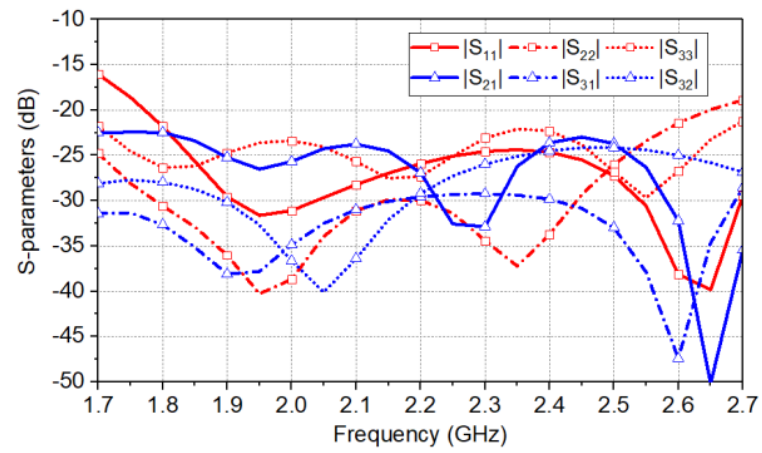

(a)

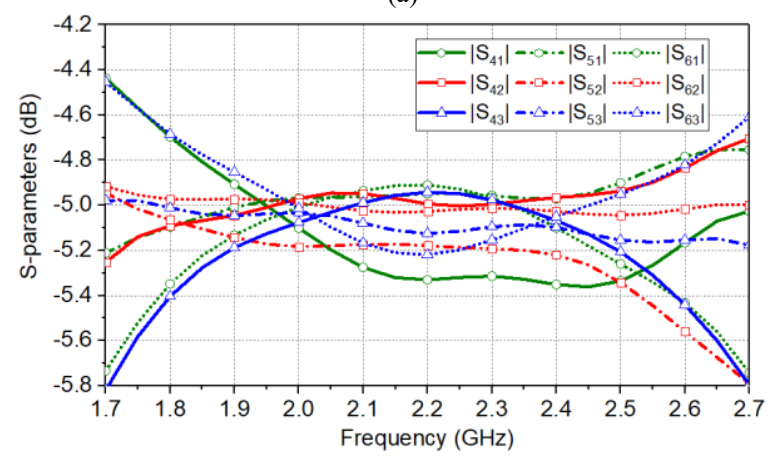

(b)

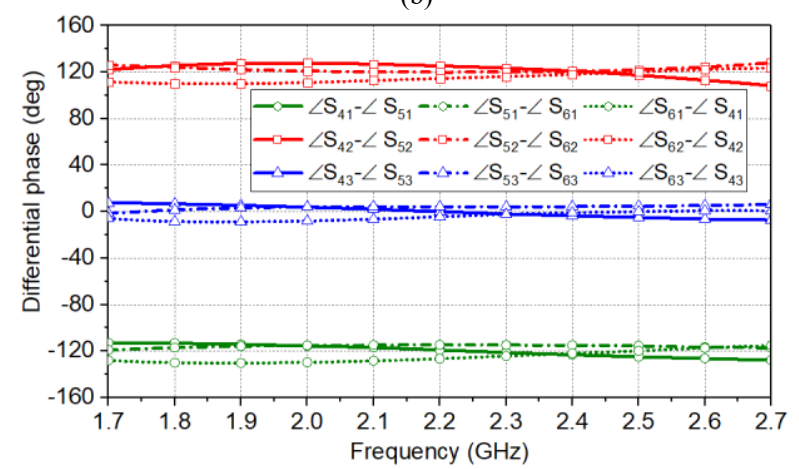

(c)

Fig. 10. Simulated S-parameters of the fabricated Butler matrix: (a) return loss and isolations; (b) transmission coefficients; (c) differential phases.

selected as $\theta_{r}=270^{\circ}$. The impedance of $Z_{1}$ and $Z_{2}$ will affect the return loss, insertion loss and phase deviation within the operating band range. In this design, to cover the wide band range and keep a minimized phase deviation, $Z_{1}$ and $Z_{2}$ are selected as $30 \Omega$ and $33 \Omega$, respectively. To verify the design, full-wave simulation is done using the stripline structure. Fig. 7 shows the $3 \mathrm{D}$ configuration of the phase shifter. It is noted that only one layer of stripline is used, which means the distances between the metal conductor and two grounds are different as $h_{1}$ and $h_{1}+h_{2}$, respectively.

Fig. 8 shows the comparison between the synthesized and full-wave simulation results of the structure. The full-wave simulation is done in the EM environment Ansys EM Desktop using the same substrate configuration as for the quadrature couplers. The optimized dimensions of the phase shifter are found to be: $l_{1}=4.9, l_{2}=5.14, l_{3}=20, l_{4}=13.9, w_{1}=$ 1.96, $w_{2}=3.6, w_{3}=1.4$, all in $\mathrm{mm}$. The full-wave simulated result demonstrates that over the $1.7 \mathrm{GHz}$ to $2.7 \mathrm{GHz}$ band, the return loss is more than $20 \mathrm{~dB}$ and insertion loss is close to 0

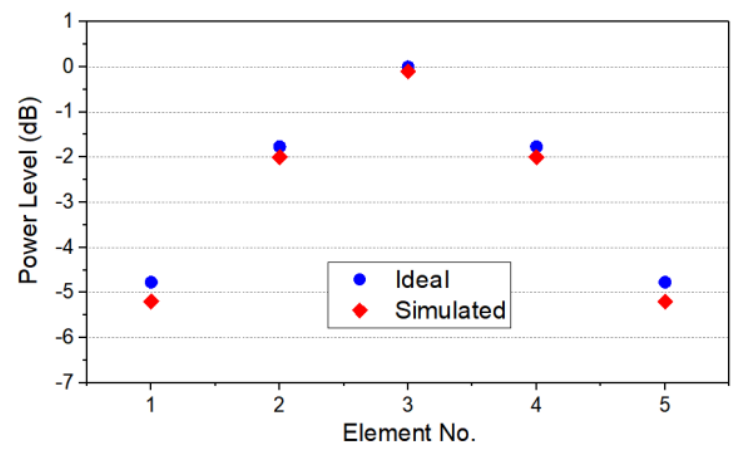

(a)

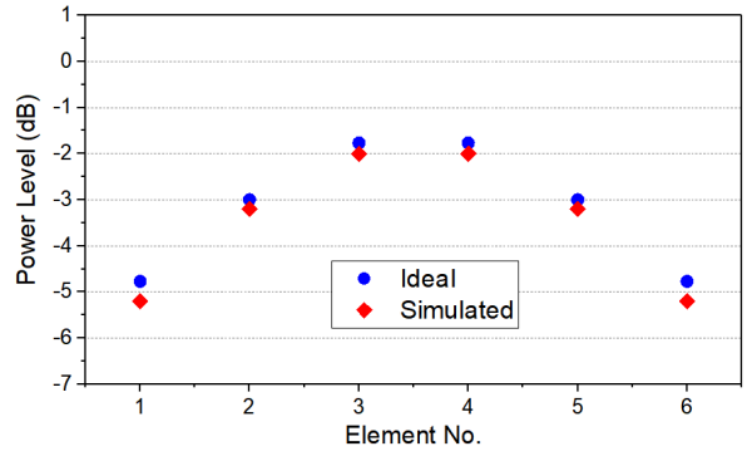

(b)

Fig. 11. Ideal and simulated power distribution for: (a) 5-element and (b) 6-element beam-forming arrays.

dB. Three resonant poles can be observed which greatly broaden the operating band. With regards to the differential phase, $\Delta \phi$ is around $90 \pm 2^{\circ}$ across the same band range, which makes it very suitable for minimizing the phase deviation of the beam-forming network. It is noted that another $180^{\circ}$ phase shifter is also required in constructing the 3-beam Butler matrix; however, since $180^{\circ}$ requires extremely small value of impedance that is not practical to realize, it is feasible to use two $90^{\circ}$ phase shift units to replace the $180^{\circ}$ one in the following design process.

\section{WIDEBAND BEAM-FORMING NETWORKS}

\section{A. Modeling and Simulation of Butler Matrices}

After the wideband quadrature couplers and phase shifters are designed, a three-beam Butler matrix can be created using the configuration shown in Fig. 2. Fig. 9(a) shows the 3D model of the Butler matrix built in the full-wave environment Ansys EM Desktop. Stripline structure using the same substrate is used. To satisfy the requirement of differential phases at each port, appropriate length delay lines are implemented as meandered lines, making the structure more compact. Two $3-\mathrm{dB}$ and one 1.77-dB quadrature couplers are used as indicated in the configuration of the beam-forming network shown in Fig. 2. Several $90^{\circ}$ phase shifters provide $90^{\circ}$ phase shift while two units are in series connection to provide $180^{\circ}$ phase shift at port 6 , which is in line with the configuration of the three-beam Butler matrix shown in Fig. 2. Specific dimensions of the structure are given in Fig. 9(b).

Fig. 10 shows the performance of the three-beam Butler matrix. As shown in Fig. 10(a), excellent matching at all ports 


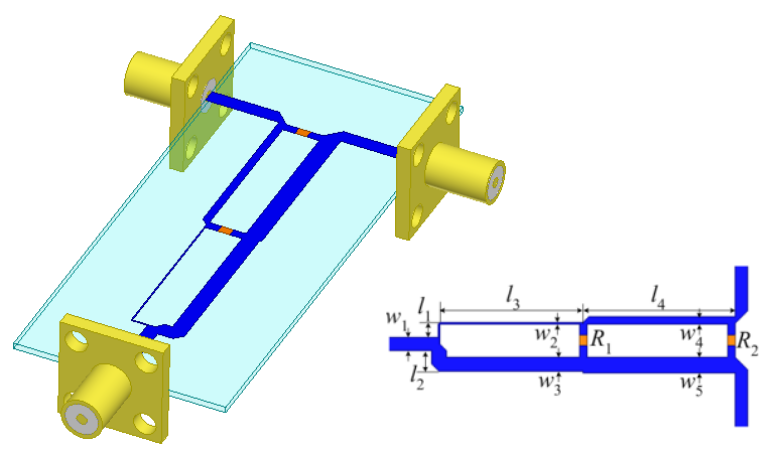

(a)

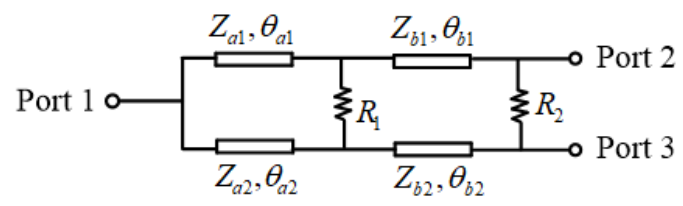

(b)

Fig. 12. Wideband Wilkinson splitters: (a) the printed circuit board realization; (b) equivalent circuit.

and isolation between input ports is obtained with $\left|S_{i i}\right|$ and $\left|S_{i j}\right|$ $(i, j=1,2$ or 3$)$ are all less than $-20 \mathrm{~dB}$ across the band. The transmission from input to output terminals are given in Fig. 10 (b), which are around -4.4 to $-5.8 \mathrm{~dB}$, and comparing well with the theoretical value of $-4.77 \mathrm{~dB}$ at each) port, the overall insertion loss is around $0.4 \mathrm{~dB}$. Fig. 10(c) shows the differential phase of all cases. To realize the multiple-beam antenna arrays, it is required to have differential phase of $-120^{\circ}, 120^{\circ}$, and $0^{\circ}$ when port 1 , port 2 and port 3 are excited. The Simulated phase deviation is around $\pm 10^{\circ}$. These results guarantee good matching, equal power, and constant phase increments for the beam-forming networks.

\section{B. Wideband Power Splitters}

To build the beam-forming networks shown in Fig. 1, equal and unequal power dividers with equal phase outputs are needed. The power distributions of the 5- and 6-element arrays are tapered as shown in Fig. 11(a) and (b). This type of tapered distribution is applied in both cases and has the effect of reducing the side-lobe levels [33]. For the 5-element array, the center element has the highest power level of $0 \mathrm{~dB}$ and it decreases to $-1.8 \mathrm{~dB}$ and $-4.8 \mathrm{~dB}$ at the two edges. For the 6-element array, the centre two elements have the highest power level of $-1.8 \mathrm{~dB}$, while the power levels at the other four elements are at $-3 \mathrm{~dB},-3 \mathrm{~dB},-4.8 \mathrm{~dB}$ and $-4.8 \mathrm{~dB}$, respectively.

Fig. 12 shows the configuration of the splitters and their equivalent circuit using ideal transmission lines. Due to the requirement for wide operating bandwidth, single-section Wilkinson power splitters are not suitable. Therefore, a two-section splitter, composed of four transmission lines $\left(Z_{a 1}, \theta_{a 1}\right),\left(Z_{a 2}, \theta_{a 2}\right),\left(Z_{b 1}, \theta_{b 1}\right)$, and $\left(Z_{b 2}, \theta_{b 2}\right)$ and two isolation resistors $\left(R_{1}, R_{2}\right)$, is used, as indicated in Fig. 12(b). For wideband performance, the electrical length of each transmission line should be a quarter- wavelength at the centre frequency. For an unequal power divider, we require $S_{11}=S_{22}$

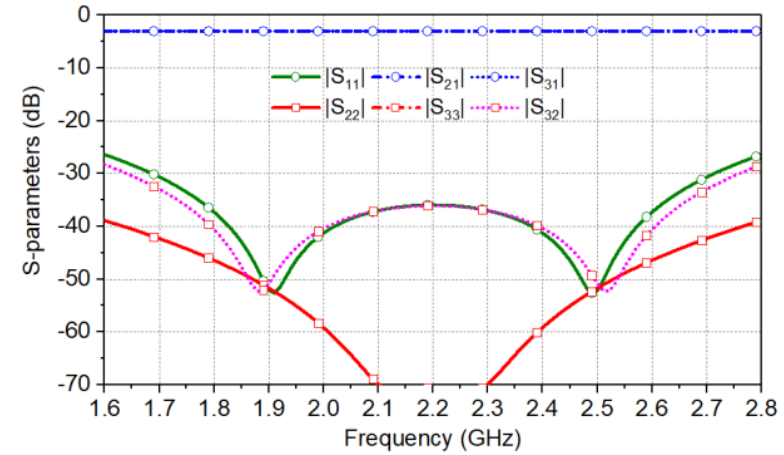

(a)

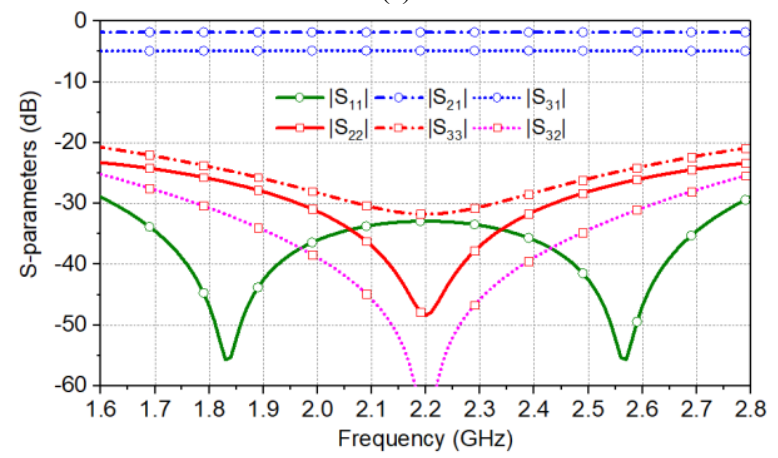

(b)

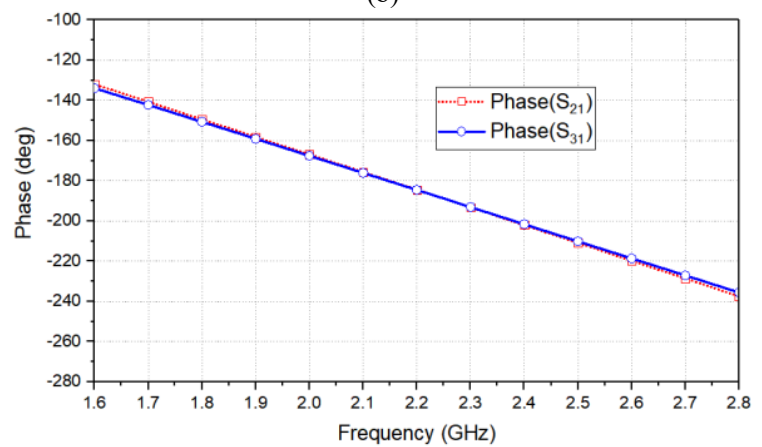

(c)

Fig. 13. Simulated (a) magnitude of $\mathrm{S}_{11}$ and $\mathrm{S}_{21}$, (b) magnitude of $\mathrm{S}_{31}$ and $\mathrm{S}_{41}$, and (c) phases of $S_{21}$ and $S_{31}$ of the wideband power splitter.

TABLE IV

RELATED PARAMETERS OF TWO POWER DIVIDERS

\begin{tabular}{cccc}
\hline \hline \multicolumn{2}{c}{$1: 1$ power divider } & \multicolumn{2}{c}{$2: 1$ power divider } \\
Parameters & Values $(\mathrm{mm})$ & Parameters & Values $(\mathrm{mm})$ \\
\hline$l_{1}$ & 1.9 & $l_{1}$ & 2.05 \\
$l_{2}$ & 1.9 & $l_{2}$ & 2.85 \\
$l_{3}$ & 19.2 & $l_{3}$ & 19.46 \\
$l_{4}$ & 20.1 & $l_{4}$ & 20.56 \\
$w_{1}$ & 1.7 & $w_{1}$ & 1.7 \\
$w_{2}$ & 0.69 & $w_{2}$ & 0.15 \\
$w_{3}$ & 0.69 & $w_{3}$ & 1.94 \\
$w_{4}$ & 1.33 & $w_{4}$ & 0.94 \\
$w_{5}$ & 1.33 & $w_{5}$ & 2.17 \\
$R_{1}$ & $100 \Omega$ & $R_{1}$ & $100 \Omega$ \\
$R_{2}$ & $200 \Omega$ & $R_{2}$ & $240 \Omega$ \\
\hline \hline
\end{tabular}

$=S_{33}=S_{32}=0$ and the amplitude ratio of outputs is $\left|S_{21}\right| /\left|S_{31}\right|=k$, and

$$
S_{21}=\frac{k}{\sqrt{k^{2}+1}}
$$




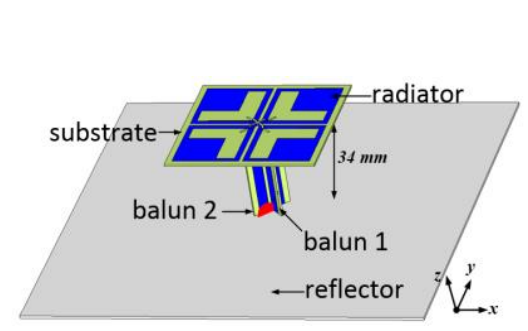

(a)

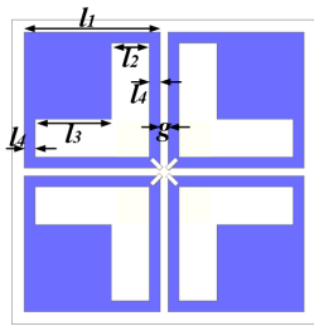

(b)

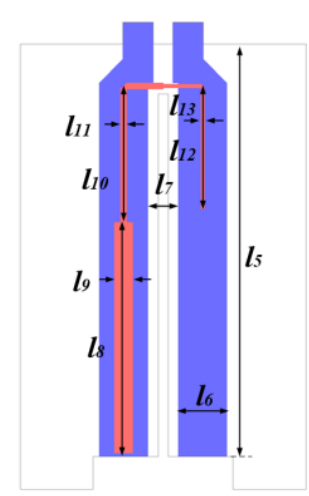

(c)

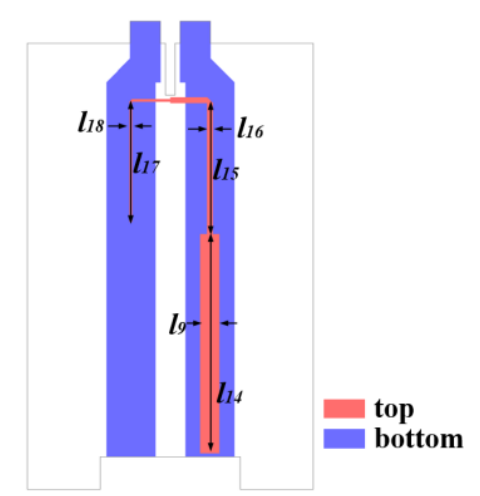

(d)
Fig. 14. Configuration of (a) the $\pm 45^{\circ}$-polarized antenna element; (b) radiator; (c) balun 1, and (d) balun 2 .

TABLE V

OPTIMIZED PARAMETERS OF THE $\pm 45^{\circ}$-POLARIZED ANTENNA ELEMENT

\begin{tabular}{cccc}
\hline \hline Parameters & Values $(\mathrm{mm})$ & Parameters & Values $(\mathrm{mm})$ \\
\hline$l_{1}$ & 25 & $l_{2}$ & 7 \\
$l_{3}$ & 14 & $l_{4}$ & 2 \\
$l_{5}$ & 34 & $l_{6}$ & 4 \\
$l_{7}$ & 2.5 & $l_{8}$ & 19 \\
$l_{9}$ & 1.55 & $l_{10}$ & 11.25 \\
$l_{11}$ & 0.5 & $l_{12}$ & 10.15 \\
$l_{13}$ & 0.3 & $l_{14}$ & 18 \\
$l_{15}$ & 11 & $l_{16}$ & 0.45 \\
$l_{17}$ & 10 & $l_{18}$ & 0.2 \\
$g$ & 1.4 & & \\
\hline \hline
\end{tabular}

$$
S_{31}=\frac{1}{\sqrt{k^{2}+1}}
$$

where $k$ represents the square root of power division ratio $k^{2}$ at two output ports. Obviously, the total output power should be equal to the input power. To obtain symmetrical power level distributions on the upper and lower lines, the following equation should be satisfied so as to avoid dissipation of power in $R_{1}$ and $R_{2}$ :

$$
k^{2}=\frac{Z_{b 1}}{Z_{a 1}}=\frac{Z_{b 2}}{Z_{a 2}}
$$

Meanwhile, a suitable selection of isolation resistors $R_{1}$ and $R_{2}$ are given by:

$$
\begin{gathered}
R_{1}=2 Z_{0} \\
R_{2}=\left(k^{2}+\frac{1}{k^{2}}\right) Z_{0}
\end{gathered}
$$

Here, two power dividers are needed - one with equal power division $\left(k^{2}=1 / 1\right)$ and one with unequal division $\left(k^{2}=2 / 1\right)$. Based on the analysis above, the S-parameters of

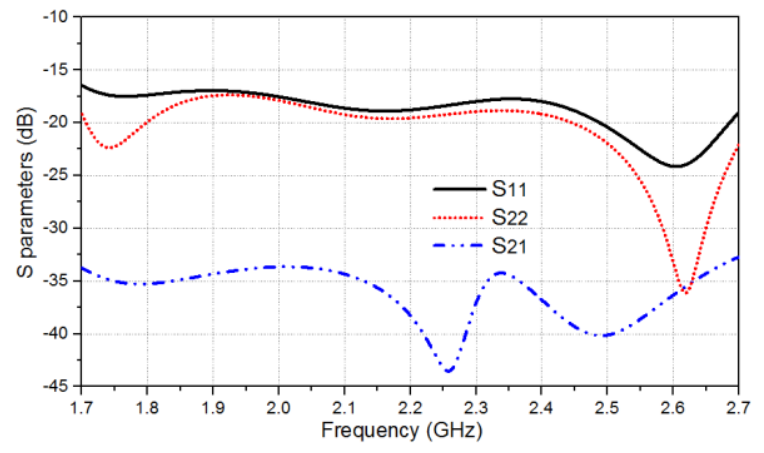

Fig. 15. Simulated S-parameters of the $\pm 45^{\circ}$-polarized single antenna element.

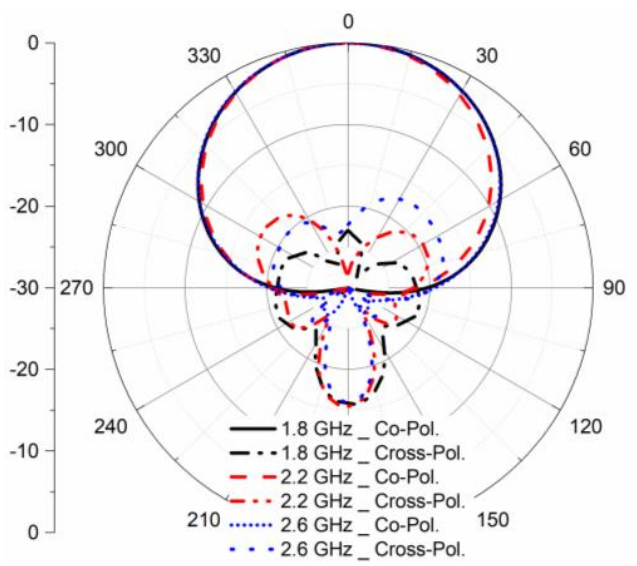

Fig. 16. Simulated radiation pattern at $1.8 \mathrm{GHz}, 2.2 \mathrm{GHz}$, and $2.6 \mathrm{GHz}$ for the $\pm 45^{\circ}$-polarized antenna element.

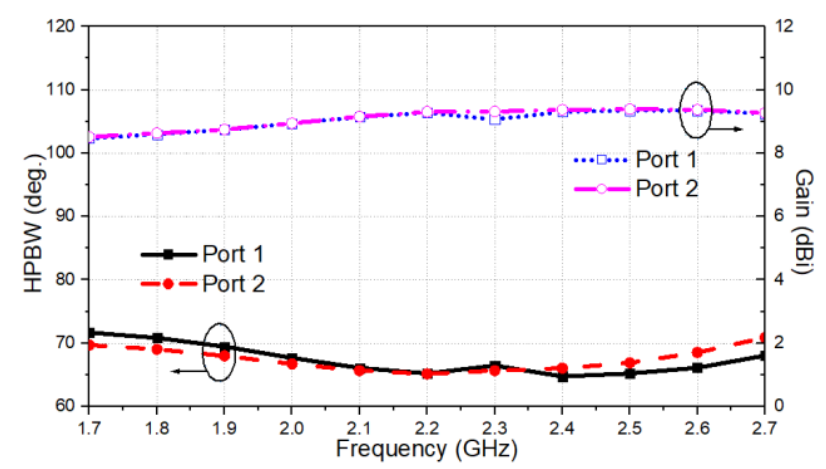

Fig. 17. Simulated HPBW and gain for the $\pm 45^{\circ}$-polarized antenna element.

two power dividers can be synthesized as given in Figs. 13(a) and (b). For the equal power splitter, it is found that $\left|S_{21}\right|=$ $\left|S_{31}\right|=-3 \mathrm{~dB}$ and $\left|S_{11}\right|,\left|S_{22}\right|,\left|S_{33}\right|$ and $\left|S_{22}\right|$ are all below $-25 \mathrm{~dB}$. For the unequal case, $\left|S_{21}\right|$ and $\left|S_{31}\right|$ are -1.8 and -4.8 $\mathrm{dB}$ respectively, with a difference of $3 \mathrm{~dB}$ indicating the power division of $2: 1$. The return loss at all ports and the isolation are greater than $20 \mathrm{~dB}$ across a wide band. The phase response of the unequal power divider is shown in Fig. 13(c). It is observed that the phase difference between two paths is smaller than $\pm 1^{\circ}$ across the whole band, which can be regarded as an in-phase power divider. An EM structure is built and the related dimensions are listed in Table IV. The simulated results agree well with the predicted ones, indicating that the design can provide proper power levels at outputs of the beam- forming networks and small reflections across such wide band range, which guarantee the overall performance of whole beamforming network when integrated in systems. 


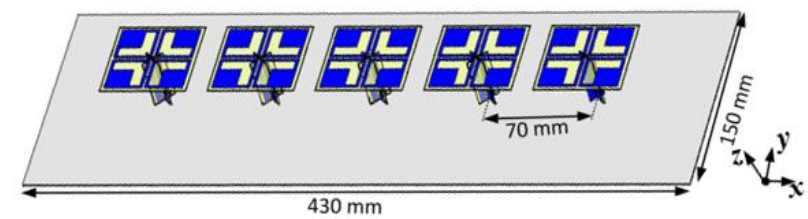

(a)

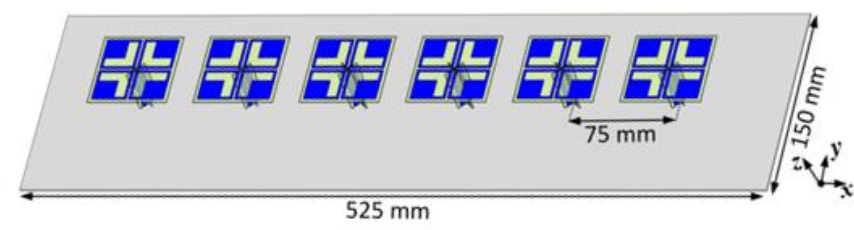

(b)

Fig. 18. Configuration of (a) 5-element antenna array, and (b) 6-element antenna array.

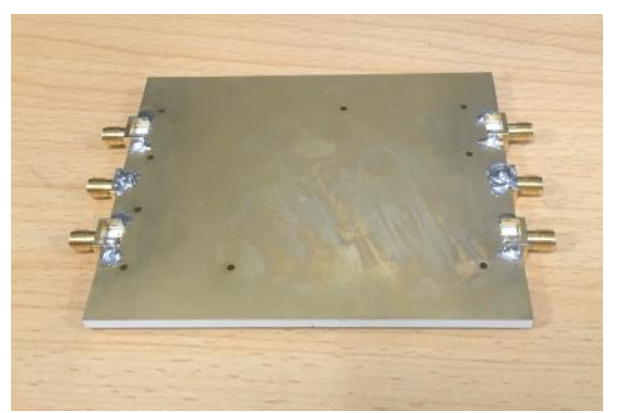

Fig. 19. Photograph of the fabricated Butler matrix prototype.

\section{RADIATING ELEMENTS AND ARRAYS}

This section shows the design of the radiating element and arrays used in the beam-forming arrays. To obtain stable patterns and minimize the effect of mutual coupling between elements, a compact structure of dual-polarized element is proposed and verified. Simulations have been conducted on a single isolated radiating element as well as on the beam-forming arrays.

\section{A. Configuration of the Radiating Element}

The configuration of the $\pm 45^{\circ}$-polarized antenna element is shown in Fig. 14. The reason for this selection is the small size of the element which permits a half-wavelength spacing. This should minimize the mutual coupling between elements. Each element consists of a square-shaped cross-dipole, two perpendicular baluns, and a flat square ground reflector. For each dipole arm, a square metal piece is added in the square loop for convenient matching and minimizing the radiator's size. The cross-dipole is printed on the top of a substrate of dielectric constant 4.3 and thickness $1.0 \mathrm{~mm}$. Two baluns are designed to excite the cross-dipole to achieve $\pm 45^{\circ}$ polarizations. The detailed design procedure with impedance matching for the wideband balun was presented in [34], and is not repeated here. The optimized dimensions of the element are listed in Table V. The element features a compact size of $51.4 \times$ $51.4 \mathrm{~mm}^{2}$.

The simulated S-parameters for a single isolated element is shown in Fig. 15. The antenna elements at both polarizations

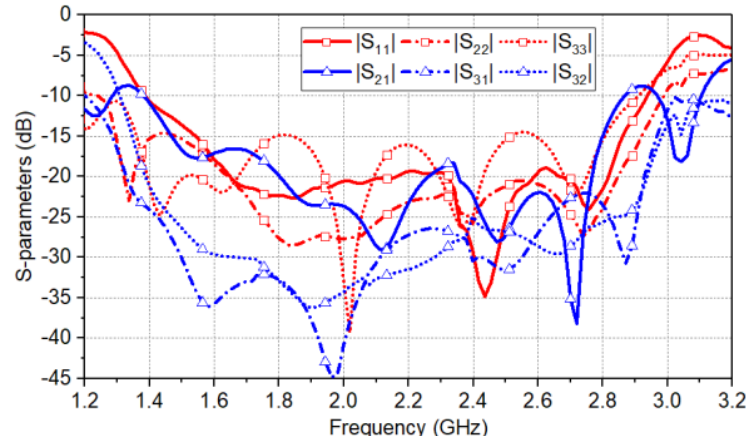

(a)

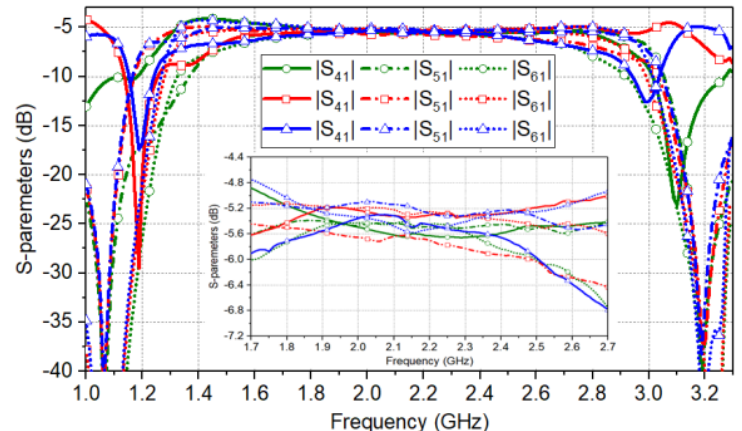

(b)

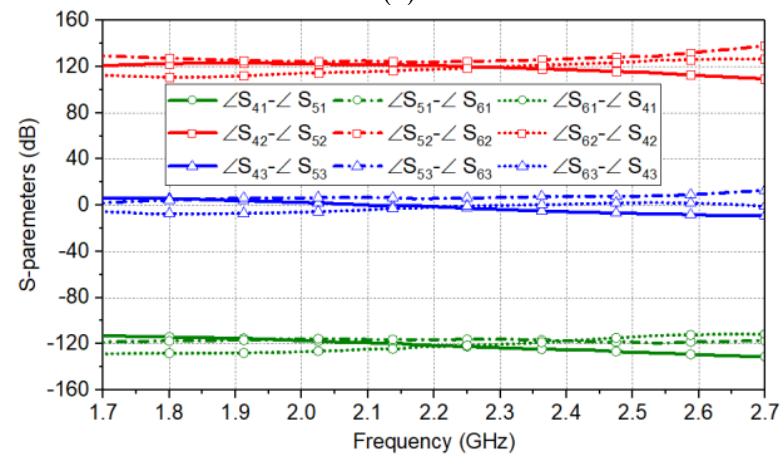

(c)

Fig. 20. Measured S-parameters of the fabricated Butler matrix: (a) return loss and isolations; (b) transmission coefficients; (c) differential phases.

are well-matched with $\left|S_{11}\right|$ and $\left|S_{22}\right|$ below $-16.5 \mathrm{~dB}$ across the band. The coupling between the two polarizations is less than $-33 \mathrm{~dB}$ across the band. The radiation pattern of the element for one polarization at $1.8 \mathrm{GHz}, 2.2 \mathrm{GHz}$, and $2.6 \mathrm{GHz}$ is given in Fig. 16, from which very stable radiation patterns can be observed, with cross-polarization level less than $-22 \mathrm{~dB}$ at boresight. The simulated horizontal HPBW and gain performance are shown in Fig. 17, which shows that the HPBW varies within $68^{\circ} \pm 2^{\circ}$ and the gain fluctuates around $9.0 \mathrm{dBi}$ from $1.7 \mathrm{GHz}$ to $2.7 \mathrm{GHz}$ for the two polarizations. The good performance of the antenna element contributes to reliable pattern of antenna arrays used in the beam-forming arrays. While the elements were designed in isolation, the performance of the array is calculated in the presence of mutual coupling.

\section{B. Arrays}

Two arrays are designed as shown in Fig 18. The spacing between the elements is of critical importance for the sidelobe level (SLL) and grating lobe level (GLL) for beams offset from boresight. The small size of the elements provides the freedom for the array arrangement. The spacing between the elements is 


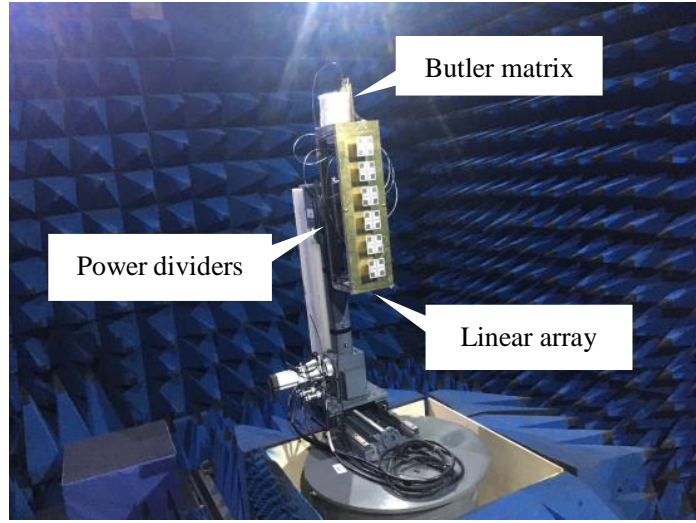

(a)

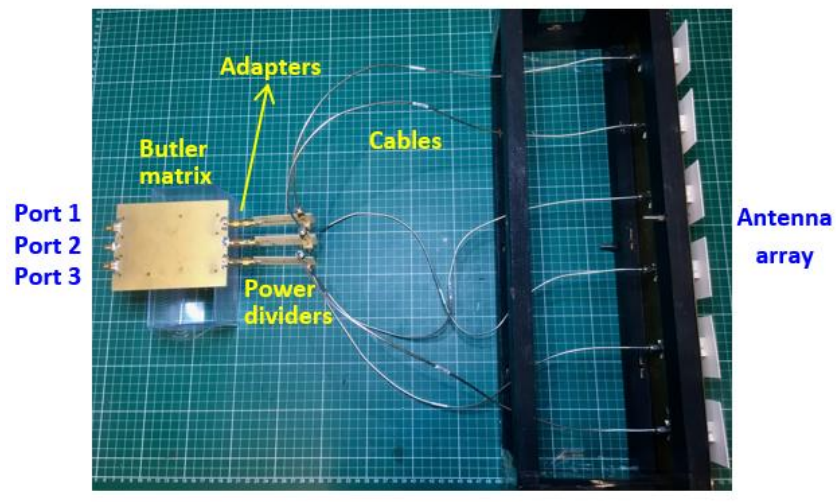

(b)

Fig. 21. The tested LTE beam-forming array including the beam-forming network and 6-element antenna array: (a) test environment; (b) overall structure.

important in arrays. For the 5-element and 6-element arrays, the distances are set to be $70 \mathrm{~mm}\left(\sim 0.50 \lambda_{0}\right)$ and $75 \mathrm{~mm}\left(\sim 0.54 \lambda_{0}\right)$, respectively. These are optimized to minimize the SLL and GLL across the band. The antenna arrays are fed from two sets of beam-forming networks for the two polarizations.

\section{EXPERIMENT RESULTS AND DISCUSSIONS}

\section{A. Experimental Results of the three-beam Butler Matrix}

Finally, a prototype of beam-forming network as well as the beam-forming array are built and tested for validation. Fig. 19 shows the fabricated prototype of the wideband three-beam Butler matrix. Since the design is implemented in stripline technology, the top and bottom layer are ground while the tracks are in the central layers and are not visible. Its overall performance is shown in Fig. 20. The measured results agree well with the predictions. As observed from Fig. 20(a), within the band, all return loss values from input ports and isolation values between any two input ports $\left(\left|S_{i i}\right|\right.$ and $\left|S_{i j}\right|, i, j=$ 1,2 or 3 ) are greater than $15 \mathrm{~dB}$. The transmission properties of $\left|S_{j i}\right|(i=1,2$ or 3 , and $j=4,5$ or 6$)$ are $-5.8 \pm 1.0 \mathrm{~dB}$, which means the insertion loss is around $1.0 \mathrm{~dB}$ for all signals. The tested differential phases at all ports are $-120^{\circ}, 120^{\circ}$, and $0^{\circ}$ when port 1 , port 2 and port 3 are excited with around $\pm 10^{\circ}$ deviation, which is the same as the simulation. These results guarantee that the three beams of the multi-beam arrays are preserved across the LTE band.

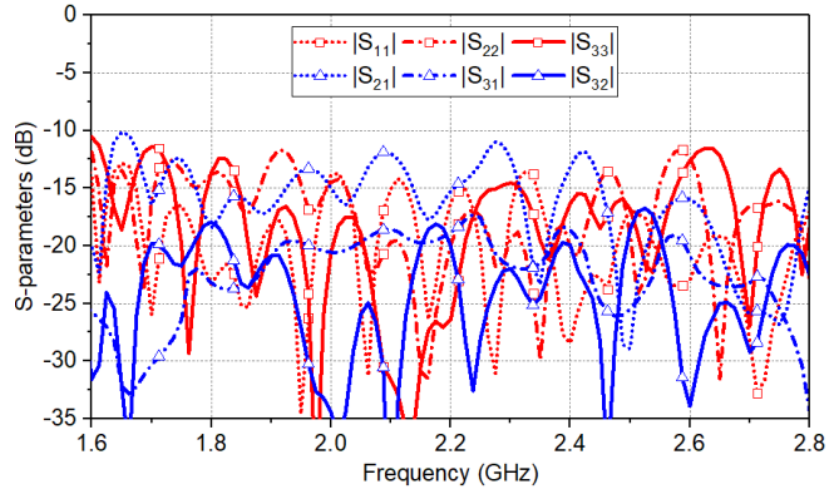

(a)

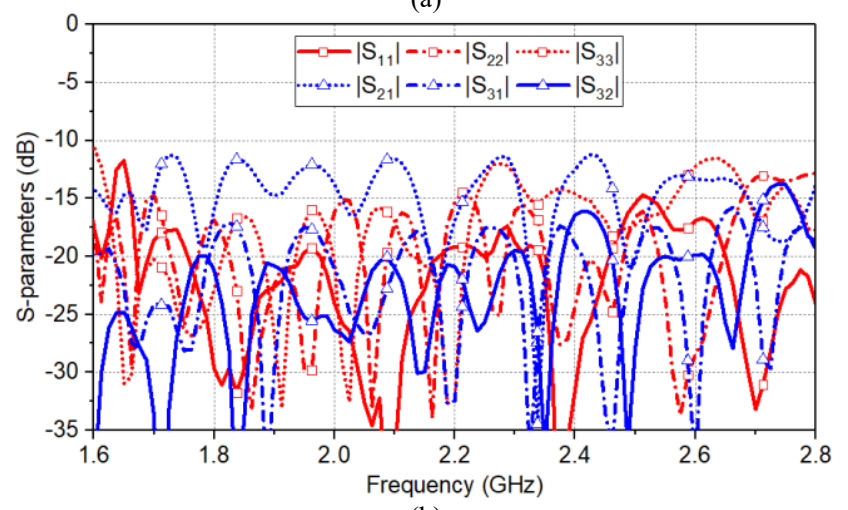

(b)

Fig. 22. Measured return loss and isolation at input ports of two beam-forming arrays: (a) using 5-element array; (b) using 6-element array.

\section{B. Experimental Results of Beam-forming Arrays}

The test environment and the overall structure of beamforming arrays and are shown in Fig. 21(a) and (b). The three-beam Butler matrix is cascaded with the three power dividers using adapters, and then the output ports of power dividers are connected with six array elements using cables. Since the two beam-forming arrays in Fig. 11 are similar in terms of structure and testing procedure, only the case of $+45^{\circ}$-pol of the 6-element array is given here. It is noted that due to the limitations of the test equipment, we rotated the array by 90 degree and tested the pattern in the elevation plane.

Fig. 22 shows the measured return losses $\left(\left|\mathrm{S}_{11}\right|,\left|\mathrm{S}_{22}\right|\right.$, and $\left.\left|\mathrm{S}_{33}\right|\right)$ and isolation $\left(\left|\mathrm{S}_{21}\right|,\left|\mathrm{S}_{31}\right|\right.$, and $\left.\left|\mathrm{S}_{32}\right|\right)$ of the two beamforming arrays comprising the beam-forming networks and antenna arrays. In both systems with 5-element or 6-element arrays, matching with return loss greater than $12.2 \mathrm{~dB}$ is achieved across the band from $1.6 \mathrm{GHz}$ to $2.8 \mathrm{GHz}$. Some reflections are caused by the cables, adapters and connectors used within the beam-forming arrays. Isolation better than 11 $\mathrm{dB}$ is obtained over the band. It is observed in Fig. 22 that more ripples are produced in the band compared with in Fig. 20(a) with only the Butler matrix. This phenomenon is due to the multiple reflections generated by the power dividers and antenna arrays.

The radiation patterns obtained from the 6-element array fed by the beam-forming network are presented in Fig. 23. Three spaced beams beam 1 , beam 2 and beam 3 are generated when port 1, port 2 and port 3 are excited, respectively. Since 


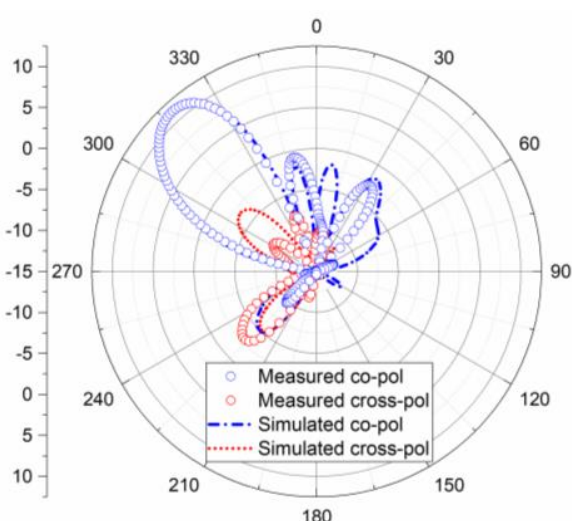

(a)

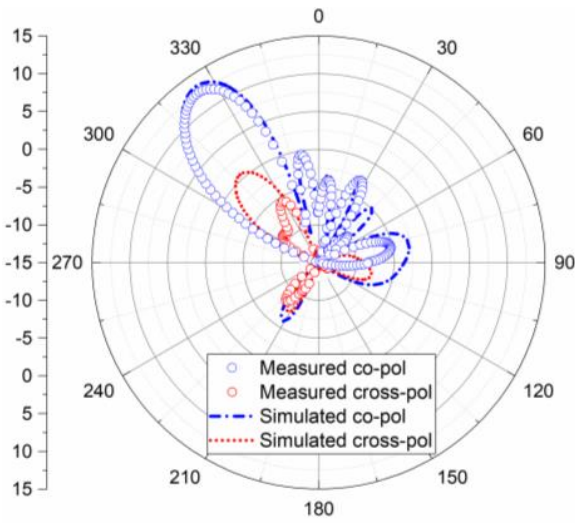

(d)

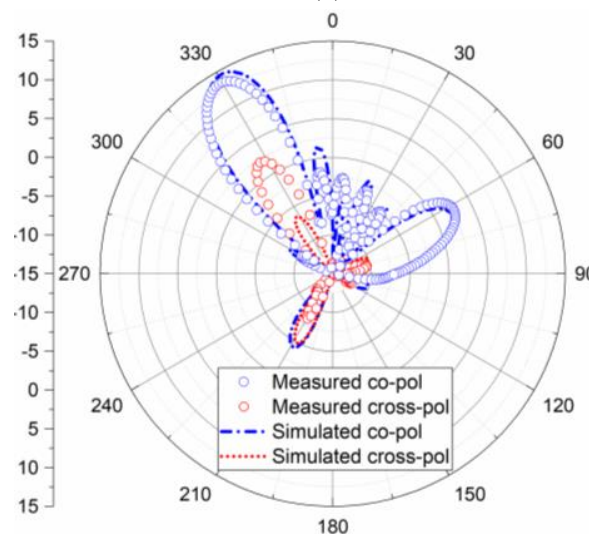

(g)

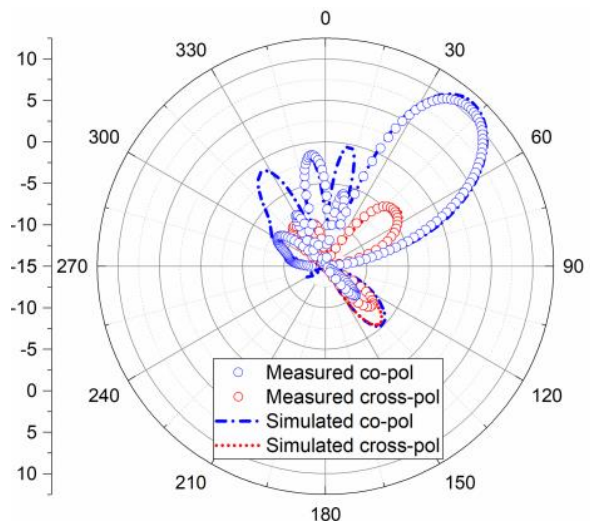

(b)

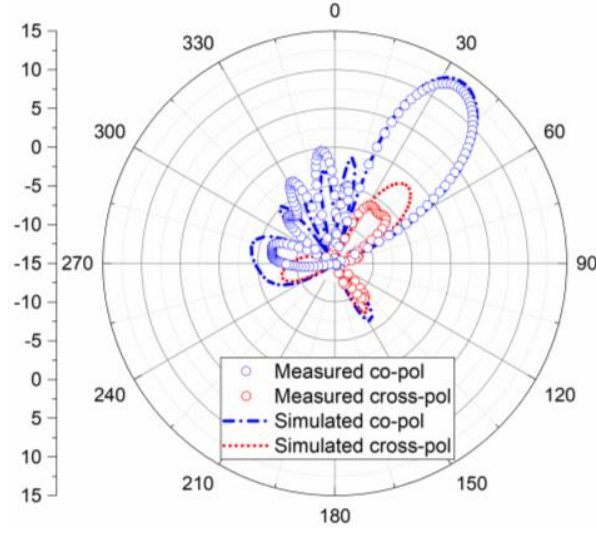

(e)

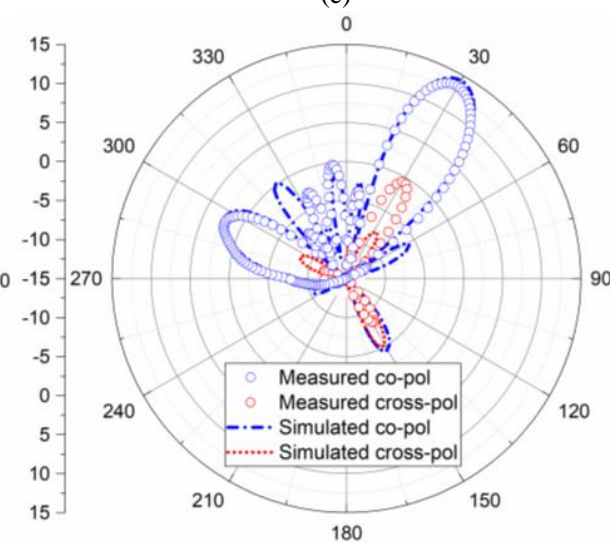

(h)

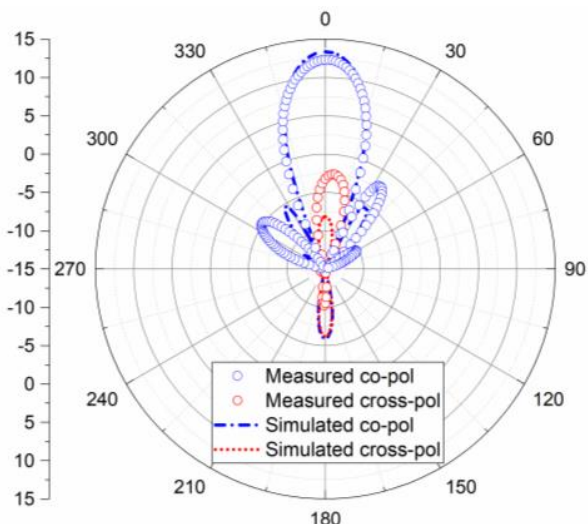

(c)

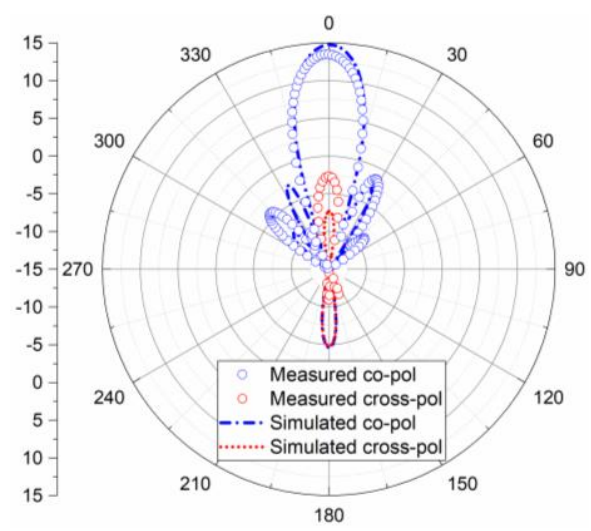

(f)

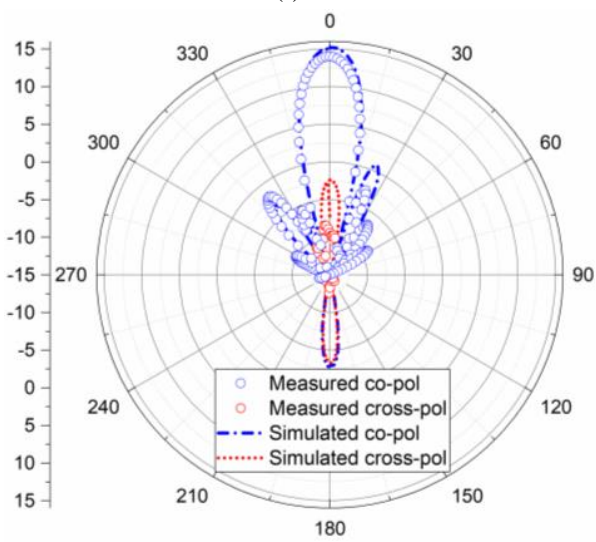

(i)

Fig. 23. Simulated radiation pattern at $1.8 \mathrm{GHz}, 2.2 \mathrm{GHz}$, and $2.6 \mathrm{GHz}$ for the 6-element antenna array fed by the beam-forming network: (a) beam 1 at $1.8 \mathrm{GHz}$; (b) beam 2 at $1.8 \mathrm{GHz}$; (c) beam 3 at $1.8 \mathrm{GHz}$; (d) beam 1 at $2.2 \mathrm{GHz}$; (e) beam 2 at $2.2 \mathrm{GHz}$; (f) beam 3 at $2.2 \mathrm{GHz}$; (g) beam 1 at $2.6 \mathrm{GHz}$; (h) beam 2 at $2.6 \mathrm{GHz}$; (i) beam 3 at $2.6 \mathrm{GHz}$.

the beams obtained using the 5-element and 6-element arrays are similar, only the patterns of the 6-element array are shown. The simulated and measured patterns are provided at three frequencies, $1.8 \mathrm{GHz}, 2.2 \mathrm{GHz}$ and $2.6 \mathrm{GHz}$. Good agreement between the simulated patterns and the measured ones is achieved. Three contiguous beams with different pointing angles are realized by the beam-forming network across the LTE band. As shown in Figs. 23(a)-(c), beams at $1.8 \mathrm{GHz}$ point at $-42^{\circ}, 42^{\circ}$ and $0^{\circ}$ when port 1 , port 2 and port 3 of the beam-forming network are excited respectively. The measured horizontal HPBW of these three states are $23^{\circ}, 23^{\circ}$ and $20^{\circ}$, which are exactly those predicted. The realized gain is around
11.8 to $13 \mathrm{dBi}$ and the SLL is below $-12 \mathrm{~dB}$. At $2.2 \mathrm{GHz}$ as shown in Figs. 23(d)-(f), the beams point at $-36^{\circ}, 36^{\circ}$ and $0^{\circ}$ and HPBWs are around $20^{\circ}, 20^{\circ}$ and $16^{\circ}$ when port 1 , port 2 and port 3 are excited. The realized measured gain is around 12.6 to $13.8 \mathrm{dBi}$, compared with the simulated value of 13 to $14.8 \mathrm{dBi}$. The SLL is below $-13 \mathrm{~dB}$ and cross-polarization level less than $-15 \mathrm{~dB}$ at the center of each beam. Figs. 23(g)-(i) display the radiation patterns at $2.6 \mathrm{GHz}$ with three beams pointing to $-30^{\circ}, 30^{\circ}$ and $0^{\circ}$, respectively. The HPBW is around $16^{\circ}, 16^{\circ}$ and $13^{\circ}$, and realized gain is 13.5 to $14.6 \mathrm{dBi}$. The SLL is below $-17 \mathrm{~dB}$ and cross-polarization level less than $-16 \mathrm{~dB}$. 
TABLE VI

OVERALl PERFormanCE OF THE 6-ELEMENT ARRAY FED BY THE BEAM-Forming NeTwORK

\begin{tabular}{|c|c|c|c|c|c|c|c|c|c|}
\hline & \multicolumn{3}{|c|}{$1.8 \mathrm{GHz}$} & \multicolumn{3}{|c|}{$2.2 \mathrm{GHz}$} & \multicolumn{3}{|c|}{$2.6 \mathrm{GHz}$} \\
\hline & Port 1 & Port 2 & Port 3 & Port 1 & Port 2 & Port 3 & Port 1 & Port 2 & Port 3 \\
\hline Beam angle & $-42^{\circ}$ & $42^{\circ}$ & $0^{\circ}$ & $-36^{\circ}$ & $36^{\circ}$ & $0^{\circ}$ & $-30^{\circ}$ & $30^{\circ}$ & $0^{\circ}$ \\
\hline HPBW & $23^{\circ}$ & $23^{\circ}$ & $20^{\circ}$ & $20^{\circ}$ & $20^{\circ}$ & $16^{\circ}$ & $16^{\circ}$ & $16^{\circ}$ & $13^{\circ}$ \\
\hline $\begin{array}{l}\text { Realized Gain } \\
\qquad(\mathrm{dBi})\end{array}$ & 12 & 11.8 & 13 & 13 & 13.2 & 14.8 & 14.8 & 14.6 & 15.1 \\
\hline $\begin{array}{l}\text { Cross-pol Level } \\
\text { (dB) }\end{array}$ & -22 & -16 & -15 & -18 & -19 & -15 & -11 & -14 & -22 \\
\hline $\begin{array}{l}\text { Side-lobe Level } \\
(\mathrm{dB})\end{array}$ & -13 & -14 & -14 & -13 & -13 & -15 & -15 & -14 & -17 \\
\hline
\end{tabular}

Table VII

Comparison Between This Work with Other Beam-forming Networks and Antenna Arrays

\begin{tabular}{|c|c|c|c|c|c|c|c|c|c|c|}
\hline Ref. & Type & Configuration & $\begin{array}{c}\mathrm{FR} \\
(\mathrm{GHz})\end{array}$ & $\begin{array}{l}\text { BW } \\
(\%)\end{array}$ & $\mathrm{IL}(\mathrm{dB})$ & $\begin{array}{l}\mathrm{RL} \\
(\mathrm{dB})\end{array}$ & $\begin{array}{l}\text { Isolation } \\
\text { (dB) }\end{array}$ & $\begin{array}{c}\text { Antenna } \\
\text { Realization }\end{array}$ & Gain (dBi) & $\begin{array}{c}\text { HPBW } \\
(\%)\end{array}$ \\
\hline [15] & $4 \times 4$ & Microstrip & $5.8-6.4$ & 9.8 & NG & -10 & -18 & Yes & $8.7-9.8$ & NG \\
\hline [16] & $8 \times 8$ & Stripline & 2.45 & NA & $2-3$ & -10 & $\mathrm{NG}$ & Yes & $7.4-10.8$ & $\mathrm{NG}$ \\
\hline [17] & $4 \times 4$ & Microstrip & $4.4-5.8$ & 23.5 & $\mathrm{NG}$ & -12 & $\mathrm{NG}$ & Yes & $\sim 7.1$ & 36 \\
\hline [19] & $4 \times 8$ & Waveguide & $58.5-62.5$ & 4 & $\mathrm{NG}$ & -10 & -10 & Yes & $25-27$ & $17-21$ \\
\hline$[20]$ & $4 \times 4$ & SIW & $25-28$ & 11.3 & $1.5-3$ & -10 & $\mathrm{NG}$ & Yes & $11.9-12.8$ & $\mathrm{NG}$ \\
\hline [21] & $4 \times 4$ & SIW & $8.5-10.6$ & 22 & $\mathrm{NG}$ & -15 & -15 & No & NA & NA \\
\hline [23] & $4 \times 4$ & Microstrip & 1 & NA & 1.75 & -18 & -15 & No & NA & NA \\
\hline$[24]$ & $4 \times 4$ & Microstrip & $0.96-1.04$ & 8 & 1.5 & -10 & -10 & Yes & $\mathrm{NG}$ & $\mathrm{NG}$ \\
\hline$[25]$ & $4 \times 4$ & $\begin{array}{l}\text { Three-layer } \\
\text { Microstrip }\end{array}$ & $1.96-3.15$ & 47 & $1.4-3.4$ & -15 & NG & Yes & NG & NG \\
\hline$[26]$ & $4 \times 4$ & Microstrip & 2.4 & NA & 1.7 & -14 & -18.2 & Yes & $7-10$ & $26-42.5$ \\
\hline$[27]$ & $3 \times 3$ & Microstrip & $5.6-6$ & 7 & $1-2.4$ & -15 & -20 & Yes & $\sim 10$ & $\mathrm{NG}$ \\
\hline [28] & $3 \times 4$ & Microstrip & $2.1-3$ & 37 & $1.5-3$ & -15 & -15 & No & NA & NA \\
\hline [29] & $3 \times 3$ & Microstrip & $1.7-2.7$ & 46 & $1-4$ & -14 & -20 & No & NA & NA \\
\hline$[30]$ & $2 \times 8$ & Microstrip & $\begin{array}{l}1.7-2.2 \\
2.4-2.8\end{array}$ & $\begin{array}{c}26 \\
15.4\end{array}$ & $\mathrm{NG}$ & -15 & -10 & Yes & $7.2-8.5$ & $\begin{array}{l}58-60 \\
80-84 \\
\end{array}$ \\
\hline $\begin{array}{l}\text { This } \\
\text { work }\end{array}$ & $\begin{array}{l}3 \times 5 \\
3 \times 6\end{array}$ & Stripline & $1.7-2.7$ & 46 & $0.5-1.5$ & -15 & -18 & Yes & $11.8-15.1$ & $16-20$ \\
\hline
\end{tabular}

\section{Discussions}

The overall performance of the beam-forming arrays is given in Table VI. As expected, as the operating frequency increases from $1.8 \mathrm{GHz}$ to $2.6 \mathrm{GHz}$, the beam-pointing angle slightly moves to the boresight, the HPBW becomes narrower, and the realized gain is slightly increased. The side-lobe level is improved as the operating frequency increases, but a grating lobe rises. These effects are mainly due to the variation of the spacing in wavelengths between elements. The variation of the pattern across the band is not significant and the beam crossover level between adjacent beams is almost constant. The achieved crossover values for the 5- and 6-element arrays are $-10 \mathrm{~dB}$ and $-15 \mathrm{~dB}$, which are exactly in line with the target. It is noted that the mutual coupling of the arrays has been included in all simulations. The patterns hardly change between 1.71 $\mathrm{GHz}$ and $1.8 \mathrm{GHz}$ as well as between $2.6 \mathrm{GHz}$ and $2.69 \mathrm{GHz}$.

Table VII shows the comparison between the performance obtained in this work and other beam-forming arrays. In this table, the overall performance of beam-forming networks (such as insertion loss, return loss, isolation) and the performance of antenna arrays (including the realized gain and half-power beamwidth) are given. From the circuit perspective, the beam-forming network in this work has excellent performance including the lowest loss, small reflection, high isolation across, and wide operating bandwidth. In order to get stable patterns, compact dual-polarized antenna arrays are developed using a compact antenna element. The antenna arrays are well matched by wideband baluns with the mutual coupling considered. The presented beam-forming network and beamforming arrays have demonstrated the largest operating bandwidth and the most stable patterns across the targeted LTE band range.

It is noted that the beam-forming network can generate three adjacent beams radiating at different angles to cover a certain area in the azimuth plane. Since the measured isolation between the beam-forming inputs is high, there is very little interference between the three beams. Therefore, 
signals targeted at different sub-sectorial regions can be transmitted at different ports of the beam-forming networks simultaneously. In this way, the function of transmitting multiple beams is realized and thus the data capacity of the communication system is tripled. It is also noted that in this work, three-beam beam-forming networks are considered because of their importance in LTE networks. The same circuit techniques are applicable to the more usual $2^{n}$-way networks where wideband operation is required. A 4-way matrix implemented with these components will have similar loss, isolation and return-loss performance.

\section{CONCLUSION}

In this paper, a novel wideband beam-forming network and two multiple beam-forming arrays for LTE base stations have been presented and verified. The designs are described in detail and are directly applicable to other configurations covering the LTE band. Thorough analysis of a beam-forming network generating three beams has been given. The design of wideband components including quadrature couplers, phase shifters and power splitters are reported. Since the stripline techniques are used throughout the whole design, the overall loss of the circuit is extremely small. To implement the function of beam-forming network, two different LTE sub-system configurations are designed and verified with antenna arrays composed of five and six $\pm 45^{\circ}$ linearly-polarized elements. Measurements have been conducted successfully on the beam-forming networks as well as on the two beam-forming arrays. The results show that the proposed design can increase system capacity by producing multiple beams in free space, which can be used in a wide range of multi-beam LTE base stations.

\section{ACKNOWLEDGMENT}

The authors would like to thank Prof. Jianquan Huang and Mr. Feng Qiu at Xiangnan University for their help with the testing of the beam-forming arrays and valuable suggestions and comments on this work.

\section{REFERENCES}

[1] H. -D. Chen, C. -Y. -D. Sim, and J. -Y. Wu, "Broadband high-gain microstrip array antennas for WiMAX base station," IEEE Trans. Antennas Propag., vol. 60, no. 8, pp. 3977-3980, Aug. 2012

[2] C. -X. Mao, S. Gao, Y. Wang, F. Qin, and Q.-X. Chu, "Multimode resonator-fed dual-polarized antenna array with enhanced bandwidth and selectivity," IEEE Trans. Antennas Propag., vol. 63, no. 12, pp. 5492-5499, Dec. 2015.

[3] C. -X. Mao et al., "An integrated filtering antenna array with high selectivity and harmonics suppression," IEEE Trans. Microw. Theory Techn., vol. 64, no. 6, pp. 1798-1805, Jun. 2016.

[4] C. Ding, H. Sun, R. W. Ziolkowski and Y. J. Guo, "Simplified tightly-coupled cross-dipole arrangement for base station applications," IEEE Access, vol. 5, pp. 27491-27503, Nov. 2017.

[5] H. Huang, Y. Liu, and S. Gong, "A broadband dual-polarized base station antenna with sturdy construction," IEEE Antennas Wireless Propag. lett., vol. 16, pp. 665-668, 2017.

[6] Y. Cui, R. Li, and H. Fu, "A broadband dual-polarized planar antenna for 2G/3G/LTE base stations," IEEE Trans. Antennas Propag., vol. 62, no. 9, pp. 4836-4840, Sept. 2014.

[7] H. Huang, Y. Liu, and S. Gong, "A novel dual-broadband and dual-polarized antenna for $2 \mathrm{G} / 3 \mathrm{G} / \mathrm{LTE}$ base stations," IEEE Trans. Antennas Propag., vol. 64, no. 9, pp. 4113-4118, Sep. 2016.
[8] H. Huang, Y. Liu, and S. Gong, "A dual-broadband dual-polarized base station antenna for $2 \mathrm{G} / 3 \mathrm{G} / 4 \mathrm{G}$ applications," IEEE Antennas Wireless Propag. lett., vol. 16, pp. 1111-1114, 2017.

[9] Y. He, Z. Pan, X. Cheng, Y. He, J. Qiao, M. M. Tentzeris, "A novel dual-band dual-polarized miniaturized and low-profile base station antenna", IEEE Trans. Antennas Propag., vol. 63, no. 12, pp. 5399-5408, Dec. 2015.

[10] Y. He, W. Tian, L. Zhang, "A novel dual-broadband dual-polarized electrical downtilt base station antenna for $2 \mathrm{G} / 3 \mathrm{G}$ applications", IEEE Access, vol. 5, pp. 15241-15249, June 2017.

[11] G. Li, H. Zhai, L. Li, C. Liang, R. Yu, and S. Liu, "AMC-loaded wideband base station antenna for indoor access point in MIMO system," IEEE Trans. Antennas Propag., vol. 63, no. 2, pp. 525-533, Feb. 2015.

[12] D. Manteuffel and R. Martens, "Compact multimode multielement antenna for indoor UWB massive MIMO," IEEE Trans. Antennas Propag., vol. 64, no. 7, pp. 2689-2697, Jul. 2016.

[13] Butler, J. and R. Lowe, "Beam-forming matrix simplifies design of electrically scanned antennas," IEEE Trans. Electron. Devices, 170$173,1961$.

[14] M. Elhefnawy and W. Ismail, "A microstrip antenna array for indoor wireless dynamic environments," IEEE Trans. Antennas Propag., vol. 57, no. 12, pp. 3998-4002, Dec. 2009.

[15] C. R. Liu, S. Q. Xiao, Y. X. Guo, M. C. Tang, Y. Y. Bai, and B. Z. Wang, "Circularly polarized beam-steering antenna array with Butler matrix network," IEEE Antennas Wireless Propag. Lett., vol. 10, pp. 1278$1281,2011$.

[16] C. C. Chang, R. H. Lee, and T. Y. Shih, "Design of a beam switching/steering butler matrix for phased array system," IEEE Trans. Antennas Propag., vol. 58, no. 2, pp. 367-374, Feb. 2010.

[17] C. Liu, S. Xiao, Y. X. Guo, Y. Y. Bai, and B. Z. Wang, "Broadband circularly polarized beam steering antenna array," IEEE Trans. Antennas Propag., vol. 61, no. 3, pp. 1475-1479, Mar. 2013.

[18] Y. J. Cheng, X. Y. Bao, and Y. X. Guo, "60-GHz LTCC miniaturized substrate integrated multibeam array antenna with multiple polarizations," IEEE Trans. Antennas Propag., vol. 61, no. 12, pp. 5958-5967, Dec. 2013.

[19] K. Tekkouk, J. Hirokawa, R. Sauleau, M. Ettorre, M. Sano, and M. Ando, "Dual-layer ridged waveguide slot array fed by a Butler matrix with sidelobe control in the $60-\mathrm{GHz}$ band," IEEE Trans. Antennas Propag., vol. 63, no. 9, pp. 3857-3867, Sep. 2015.

[20] A. B. Guntupalli, T. Djerafi, and K. Wu, "Two-dimensional scanning antenna array driven by integrated waveguide phase shifter," IEEE Trans. Antennas Propag., vol. 62, no. 3, pp. 1117-1124, Mar. 2014.

[21] S. Karamzadeh, V. Rafii, M. Kartal, and B. S. Virdee, "Compact and broadband $4 \times 4$ SIW Butler matrix with phase and magnitude error reduction," IEEE Microw. Wireless Compon. Lett., vol. 25, no. 12, pp. 772-774, Dec. 2015.

[22] J. W. Lian, Y. L. Ban, Q. L. Yang, B. Fu, Z. F. Yu and L. K. Sun, "Planar millimeter-wave 2-D beam-scanning multibeam array antenna fed by compact SIW beam-forming network" IEEE Trans. Antennas Propag., vol. 66, no. 3, pp. 1299-1310, Mar. 2018.

[23] Y. Jeong and T. W. Kim, "Design and analysis of swapped port coupler and its application in a miniaturized Butler matrix," IEEE Trans. Microw. Theory Techn., vol. 58, no. 4, pp. 764-770, Apr. 2010.

[24] E. Gandini, M. Ettorre, R. Sauleau, and A. Grbic, "A lumped-element unit cell for beam-forming networks and its application to a miniaturized Butler matrix," IEEE Trans. Microw. Theory Tech., vol. 61, no. 4, pp. 1477-1487, Apr. 2013.

[25] T.-H. Lin, S.-K. Hsu, and T.-L. Wu, "Bandwidth enhancement of $4 \times 4$ Butler matrix using broadband forward-wave directional coupler and phase difference compensation," IEEE Trans. Microw. Theory Techn., vol. 61, no. 12, pp. 4099-4109, Dec. 2013.

[26] H. N. Chu, and T. -G. Ma, "An extended $4 \times 4$ Butler matrix with enhanced beam controllability and widened spatial coverage", IEEE Trans. Microw. Theory Tech., vol. 66, no.34, pp. 1301-1311, Mar. 2018.

[27] K. Ding, X. Fang, Y. Wang and A. Chen, "Printed dual-layer three way directional coupler utilized as $3 \times 3$ beamforming network for orthogonal three-beam antenna array," IEEE Antennas and Wireless Propagation Letters, vol.13, pp.911-914, 2014.

[28] H. Nachouane, A. Najid, A. Tribak, and F. Riouch, "Wideband $3 \times 4$ Butler matrix using Wilkinson divider for MIMO applications", 2014 International Conference on Next Generation Networks and Services (NGNS), pp. 101-105, 2014. 
[29] J. P. Wang and W.-J. Chen, "Two-layer three beam generation matrix for broadband beamforming with microstrip," IEEE International Symposium on Antennas and Propagation (AP-S), July 2017.

[30] X. -Y. Zhang, D. Xue, L. -H. Ye, Y. -M. Pan, and Y. Zhang, "Compact dual-band dual-polarized interleaved two-beam array with stable radiation pattern based on filtering elements," IEEE Trans. Antennas Propag., vol. 65, no. 9, pp. 4566-4575, Sep. 2017.

[31] D. M. Pozar, Microwave Engineering, 2nd ed: Wiley, 1997.

[32] K. C. Gupta, R. Garg, I. Bahl, and P. Bhartia, Microstrip Lines and Slotlines, 2nd ed. Norwood, MA: Artech House, 1996.

[33] S. Gruszczynski, K. Wincza, and K. Sachse, "Reduced sidelobe four beam $N$-element antenna arrays fed by $4 \times \mathrm{N}$ butler matrices," IEEE Antennas Wireless Propag. Lett., vol. 5, no. 1, pp. 430-434, Dec. 2006.

[34] H. Sun, C. Ding, B. Jones and Y. J. Guo, "A wideband base station antenna element with stable radiation pattern and reduced beam squint," IEEE Access, vol. 5, pp. 23022-23031, Oct. 2017. 